\title{
The role of tissue microstructure and water exchange in biophysical modelling of diffusion in white matter
}

\author{
Markus Nilsson • Danielle van Westen • \\ Freddy Ståhlberg • Pia C. Sundgren • \\ Jimmy Lätt
}

Received: 3 September 2012/Revised: 28 January 2013/Accepted: 1 February 2013/Published online: 27 February 2013

(C) The Author(s) 2013. This article is published with open access at Springerlink.com

\begin{abstract}
Biophysical models that describe the outcome of white matter diffusion MRI experiments have various degrees of complexity. While the simplest models assume equal-sized and parallel axons, more elaborate ones may include distributions of axon diameters and axonal orientation dispersions. These microstructural features can be inferred from diffusion-weighted signal attenuation curves by solving an inverse problem, validated in several Monte Carlo simulation studies. Model development has been paralleled by microscopy studies of the microstructure of excised and fixed nerves, confirming that axon diameter estimates from diffusion measurements agree with those from microscopy. However, results obtained in vivo are less conclusive. For example, the amount of slowly diffusing water is lower than expected, and the diffusionencoded signal is apparently insensitive to diffusion time variations, contrary to what may be expected. Recent understandings of the resolution limit in diffusion MRI, the
\end{abstract}

\footnotetext{
M. Nilsson · F. Ståhlberg

Department of Medical Radiation Physics, Lund University, Lund, Sweden

M. Nilsson ( $\square)$

LBIC, Lund University Bioimaging Center, Lund University, 221 85, Lund, Sweden

e-mail: markus.nilsson@med.lu.se

D. van Westen · F. Ståhlberg · P. C. Sundgren

Department of Diagnostic Radiology, Lund University,

Lund, Sweden

J. Lätt

Center for Medical Imaging and Physiology,

Skåne University Hospital, Lund, Sweden
}

rate of water exchange, and the presence of microscopic axonal undulation and axonal orientation dispersions may, however, explain such apparent contradictions. Knowledge of the effects of biophysical mechanisms on water diffusion in tissue can be used to predict the outcome of diffusion tensor imaging (DTI) and of diffusion kurtosis imaging (DKI) studies. Alterations of DTI or DKI parameters found in studies of pathologies such as ischemic stroke can thus be compared with those predicted by modelling. Observations in agreement with the predictions strengthen the credibility of biophysical models; those in disagreement could provide clues of how to improve them. DKI is particularly suited for this purpose; it is performed using higher $b$-values than DTI, and thus carries more information about the tissue microstructure. The purpose of this review is to provide an update on the current understanding of how various properties of the tissue microstructure and the rate of water exchange between microenvironments are reflected in diffusion MRI measurements. We focus on the use of biophysical models for extracting tissue-specific parameters from data obtained with single PGSE sequences on clinical MRI scanners, but results obtained with animal MRI scanners are also considered. While modelling of white matter is the central theme, experiments on model systems that highlight important aspects of the biophysical models are also reviewed.

Keywords Diffusion weighted imaging - Microstructure · Exchange $\cdot$ Permeability $\cdot$ White matter
Abbreviations
ADC Apparent diffusion coefficient
MD Mean diffusivity
FA Fraction anisotropy 
List of symbols related to the diffusion MRI experiment

$g, \mathbf{n}, \delta, \Delta \quad$ Amplitude, direction and duration of diffusion encoding gradients, and the time between their leading edges

$t_{\mathrm{d}} \quad$ Diffusion time

$b, q \quad$ The magnitude of diffusion encoding, with $b=(2 \pi q)^{2} \cdot t_{\mathrm{d}}$

List of model symbols, used with subscripts $\boldsymbol{j}$

$S_{\mathrm{j}} \quad$ Intensity of diffusion encoded signal

$f_{\mathrm{j}} \quad$ Signal fraction

$D_{\mathrm{j}} \quad$ ADC of a component

$\mathrm{RD}_{\mathrm{j}}$ Radial diffusivity, i.e. $\mathrm{ADC}$ in the direction perpendicular to a nerve

$\mathrm{AD}_{\mathrm{j}}$ Axial diffusivity, i.e. $\mathrm{ADC}$ in the direction parallel with a nerve

$\mathrm{RS}_{\mathrm{j}} \quad$ Radial MRI signal intensity, i.e. $S$ obtained with diffusion encoding in a direction perpendicular to the nerve

\section{Subscripts j}

$\mathrm{r} / \mathrm{h} \quad$ Restricted/hindered diffusion component

$\mathrm{f} / \mathrm{s} \quad$ Fast/slow diffusion component

\section{Other model symbols}

$d$

$g$

$D_{\text {intra }}$

$D_{\text {bulk }}$

$\mathbf{u}, \theta, \psi$

$\alpha, \beta$

$k, \tau_{\mathrm{i}}$

$P_{\mathrm{d}}$

$A / V$

$\lambda$

$v_{\text {extra }}, v_{\text {axon }}, v_{\text {myelin }}$

$w, L$
Axon diameter

Ratio between inner and outer axon diameter

Intracellular or intra-axonal diffusion coefficient

Diffusion coefficient of the bulk medium

Direction of the nerve, specified by polar and azimuthal angles

Symbols relating $\delta$ and $\Delta$ to $d$ and

$D_{\text {intra }}$

Exchange rate and intracellular

exchange time

Diffusional membrane permeability

Area to volume ratio

Tortuosity of the extracellular space

Volume fractions of the extracellular, intra-axonal and myelin spaces, respectively

Width of nodes of Ranvier, and internode length

\section{Introduction}

The diffusion MRI experiment uses magnetic field gradients to label spins, as described pedagogically elsewhere $[1,2]$. The most common design of the experiment is based on the pulsed-gradient spin-echo (PGSE) sequence, introduced by Stejskal and Tanner in 1965 [3]. Today, diffusion MRI is widely used in both neuroscience and for clinical applications, but already in 1965 Stejskal realised the technique's potential for studying tissue: "living cells form a class of colloidal particles which should exhibit restricted diffusion of the substances confined within the cell walls" [4]. In addition to conventional experiments using a single pair of diffusion encoding gradients, the use of double gradient pairs for microstructural imaging has also been suggested [5, 6]. Such double pulsed-field gradient (d-PFG) experiments were later employed for investigations of microscopic anisotropy [7-10], estimation of compartment sizes [10,11], and increasing the sensitivity to water exchange [12, 13]. Investigations using oscillating gradient waveforms represent another class of diffusion experiments, capable of exploring diffusion at very short diffusion times [14-17]. Non-conventional gradient waveforms have also been investigated [18].

Inferring information about the microstructure of tissue from the diffusion MRI experiment is an inverse problem, where models of the outcome of the experiment are fitted to the data acquired. The models describe the diffusionweighted signal $S$ for some experimental parameters, given the model parameters. Biophysical models of diffusion in white matter express $S$ directly in terms of model parameters capturing tissue properties such as the axon diameter $d$ and the fraction of water restricted in the intra-axonal space $f_{\mathrm{r}}$. Accurate quantification of the tissue properties requires the diffusion MRI experiment to be repeated several times with maximally varying experimental settings. This is typically achieved by the use of low- and high-diffusion sensitisation (high $b$-values), and long and short diffusion times [19]. Examples of biophysical models are the CHARMED and AxCaliber models [20, 21], and other similar models $[19,22,23]$. Phenomenological models, such as the diffusion tensor model used in diffusion tensor imaging (DTI) [24], kurtosis or generalized tensor model [25] used in diffusion kurtosis imaging (DKI) $[26,27]$, the stretched exponential model [28], and the ADC distribution model [29] also exist. Phenomenological models may show a high sensitivity for detecting alterations in the characteristics of the water diffusion, but do not assign the alterations to specific features of the tissue microstructure without further assumptions [30]. In addition to the phenomenological models, model-free approaches such as $q$-space analysis also exist, but they may be too sensitive to variations in experimental parameters to be useful in the analysis of data acquired with clinical MRI scanners $[31,32]$. Given that the assumptions used when deriving biophysical models are valid, these models have the potential to increase the specificity of diffusion MRI by assigning alterations in the water diffusion characteristics to specific features of the tissue microstructure. 
Modelling of water diffusion in tissue requires knowledge of the various microscopic environments in which the water molecules are located (Fig. 1a), since the properties of those environments impact the diffusion-encoded MRI signal. The glial cells are the most numerous cell type in the human brain, but these cells are small and thus constitute less than half of the human brain volume [33, 34]. For modelling of white matter diffusion, the most important structure is instead the axon [35]. The majority of vertebrate axons with diameters above $0.2 \mu \mathrm{m}$ are myelinated, i.e., surrounded by a fatty sheath, although unmyelinated axons may have diameters of up to $1.8 \mu \mathrm{m}$ [33]. In the human corpus callosum and other structures in the brain, most myelinated axons have diameters below $3 \mu \mathrm{m}[36,37]$. Axons in the spinal cord and in peripheral nerves are generally larger than in the brain. For example, axons are between 3 and $9 \mu \mathrm{m}$ wide in the mouse sciatic nerve [38], compared to 0.2 and $1.0 \mu \mathrm{m}$ in the mouse corpus callosum [39]. Axons are also characterized by the ratio between their diameter and the outer diameter of the myelin sheath ( $g$-ratio; Fig. 1b). The value of $g$ is normally in the range $0.5-0.9$, but varies as a function of age $[33,40$,

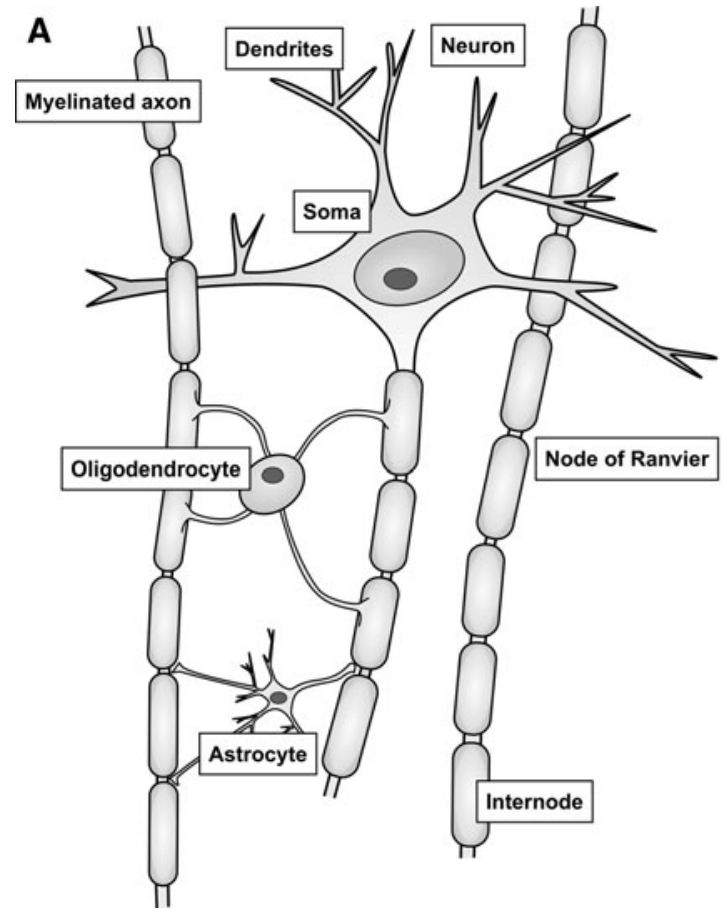

Fig. 1 Drawing of the cell components in neural tissue (a) and myelin sheath structure (b), modified from Edgar and Griffiths [33]. a The cell body of the neuron, mainly found in grey matter, is also called the soma, from which several short dendrites and a one long axon extend. Some axons are encapsulated by myelin sheaths, which wrap around the axon like a balloon around a stick. The sheaths are extensions of oligodendrocytes. These generally form myelin sheaths around several axons. Narrow regions that are called nodes of Ranvier separate the sheaths. At these nodes, the axon membrane is exposed to
41]. A value of $\exp (-1 / 2) \approx 0.6$ is optimal from a electrical conduction perspective [42]. Another important structural feature of axons are the so-called nodes of Ranvier, at which the axonal membrane (axolemma) is exposed to the extracellular space at gaps that are $0.8-1.1 \mu \mathrm{m}$ wide (Fig. 1) [43]. The distance between the nodes $(L)$ is between $100 \mu \mathrm{m}$ and $2 \mathrm{~mm}$, and increases with the axon diameter. Functionally, myelination, increased diameters and longer internode distances all contribute to increased signal transmission velocities in the axons [41, $42,44]$, at the expense of the amount of energy required per transmission [45]. Finally, some axons display a wave-like undulating course, which allow nerves to stretch during motion, such as eye movement and locomotion, without being damaged [46]. Axonal undulation is found generally in extra-cranial white matter, but is also present intracranially, for example, in the optic nerve [47, 48].

Water-channel proteins, so called aquaporins (AQP), represent another factor that may influence water diffusion in brain tissue [49]. These proteins are embedded in the cell membranes, increasing their permeability to water. The function of $\mathrm{AQP}$ in the healthy brain is only partially

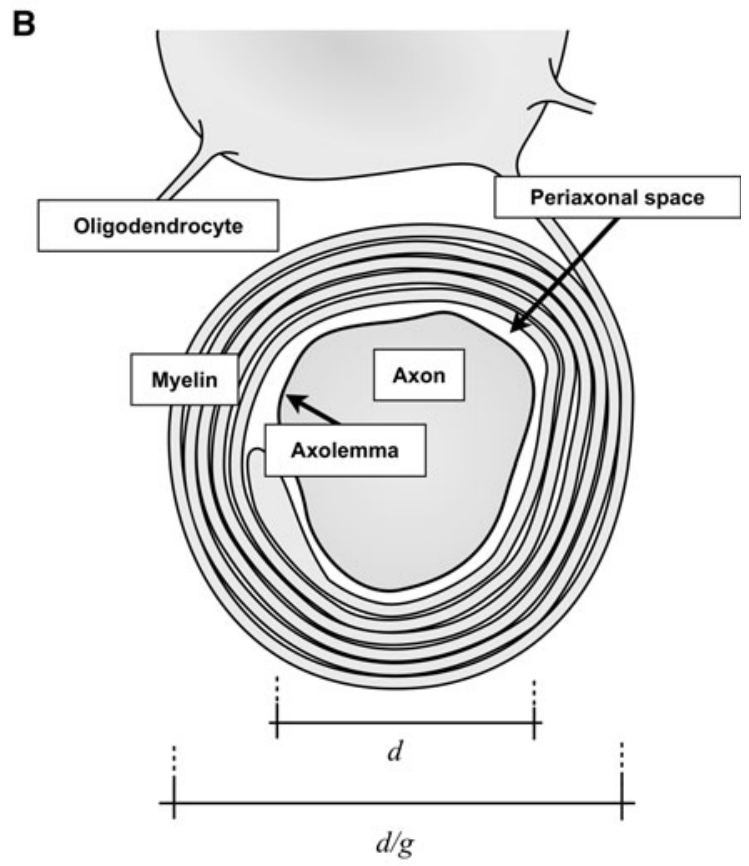

the extracellular space. The segment between two nodes is called an internode. White matter also contains star-shaped glial cells called astrocytes. These support axons, for instance by regulating the extracellular ion concentration. b The ratio between the axon diameter $d$ and the total axon diameter including the myelin is given by the $g$ ratio. A small space exists between the axolemma and the inner part of the myelin sheath, called the periaxonal space, which is approximately $15 \mathrm{~nm}$ wide and filled with extracellular fluid 
understood [50], but the channels are known to control water movement into and out of the brain in cells located at the border between brain parenchyma and major fluid compartments. They also facilitate astrocyte migration and alter neuronal activity. The expression of AQP can be altered in disease, for example, in brain oedema where the astrocytic AQP expression is upregulated. Tumours that upregulate AQP expression may also be more aggressive and it has been proposed that AQP inhibitors may slow tumour growth [50]. Aquaporins are thus attractive targets for the development of novel drug therapies [51]. Methods capable of detecting and quantifying alterations of the membrane permeability may thus find clinical use.

Understandably, neural tissue is more complex than what can be captured in relatively simple biophysical models. Estimates of biophysical model parameters should thus be compared to estimates acquired using gold-standard techniques. Obtaining reliable information regarding the three-dimensional structure of tissue and the membrane permeability in live tissue is difficult, however. Simulations and numerical methods provide an alternative for investigation of model performance in well-controlled conditions. Such understanding improves the interpretation of experiments performed in vivo or in excised nerves and cell suspensions. The purpose of this review is to provide an overview of the various components used to build biophysical models of diffusion in white matter, and to review their applicability based on simulation studies. Agreement and disagreement between model predictions and results obtained in model systems such as excised nerves and cell suspensions are also discussed. Finally, the implications of the topics discussed are considered for in vivo measurements and the clinically relevant application of ischaemic stroke.

\section{Model construction and simulation-based validation}

The goal of this section is to describe models that predict the diffusion-encoded signal in white matter. We start from the very minimal model of diffusion in white matter, and gradually extend the model to include effects of variable axon diameter, axon diameter distribution, orientation dispersion and compartmental exchange. The biological rationale for each extension is provided, along with results from simulation studies that characterize the accuracy and precision in estimates obtained with the models.

The three experimental parameters that control the diffusion weighting in a PGSE experiment are the duration and time between the onset of the diffusion-encoding gradients, denoted $\delta$ and $\Delta$, respectively, and the magnetic field gradient $\mathbf{g}$. Together, these parameters define the wave-vector $\mathbf{q}$ according to $\mathbf{q}=(\gamma / 2 \pi) \delta \mathbf{g}$, where $\gamma$ is the gyromagnetic ratio. The diffusion-sensitisation factor $b$ is given by $b=(2 \pi q)^{2} \cdot t_{\mathrm{d}}$, where $q=|\mathbf{q}|$ and the diffusion time $t_{\mathrm{d}}$ is defined by $t_{\mathrm{d}}=\Delta-\delta / 3$, assuming that the rise times of the gradients are much shorter than $\delta$. We will use the variables $b, t_{\mathrm{d}}$ and $\delta$ as the experimental parameters relevant for the model outcomes, although other triplets, such as $q, \Delta$, and $\delta$, would work equally well.

The very minimal model

Biophysical modelling of diffusion in white matter start by describing the MR signal by two components, of which one has hindered diffusion (subscript h) and the other restricted diffusion (subscript r), according to [20]

$S=S_{0}\left(f_{\mathrm{h}} S_{\mathrm{h}}+f_{\mathrm{r}} S_{\mathrm{r}}\right)$,

where $f_{\mathrm{h}}$ and $f_{\mathrm{r}}=1-f_{\mathrm{h}}$ are the signal fractions of the hindered and restricted components, respectively. Under the idealised conditions present in simulations, these components represent extracellular and intracellular water. In complex neural tissue, this assignment may only be conditionally valid, as will be discussed. Also note that the signal fractions denote the relative water populations after considering effects of potentially differing longitudinal and transversal relaxation rates in the components. The signal of $S_{\mathrm{h}}$ and $S_{\mathrm{r}}$ in Eq. 1 is given by

$S_{\mathrm{h}}=\exp \left(-b D_{\mathrm{h}}\right)$ and $S_{\mathrm{r}}=\exp \left(-b D_{\mathrm{r}}\right)$.

This model thus contains four parameters : $S_{0}, f_{\mathrm{r}}, D_{\mathrm{h}}$ and $D_{\mathrm{r}}$. Without further assumptions, this model is identical to the biexponential model $[52,53]$. Note that $D_{\mathrm{h}}$ and $D_{\mathrm{r}}$ are not bulk diffusion coefficients, but rather apparent diffusion coefficients (ADCs) that are influenced by the experimental parameters and properties of the tissue.

To model the anisotropic diffusion in white matter [54], we assume that the diffusion coefficient in white matter is cylindrically symmetric along the main axis of the nerve [22], represented by the vector $\mathbf{u}$. We may thus decompose $D_{\mathrm{h}}$ and $D_{\mathrm{r}}$ into axial and radial diffusivities, denoted $\mathrm{AD}_{\mathrm{h}} / \mathrm{RD}_{\mathrm{h}}$ for the hindered component and $\mathrm{AD}_{\mathrm{r}} / \mathrm{RD}_{\mathrm{r}}$ for the restricted component. The decomposition is identical for the hindered and restricted component, and given by [20]

$D_{\mathrm{h} / \mathrm{r}}=(\mathbf{n} \cdot \mathbf{u})^{2} \mathrm{AD}_{\mathrm{h} / \mathrm{r}}+\left(1-(\mathbf{n} \cdot \mathbf{u})^{2}\right) \mathrm{RD}_{\mathrm{h} / \mathrm{r}}$,

where $\mathbf{n}$ is the diffusion encoding direction and $\mathbf{u}$ is specified by polar and azimuthal angles $\theta$ and $\psi$. In order to specify the very minimal model of diffusion in white matter, we make two assumptions. First, we assume that the axial diffusivity is identical in both components $\left(\mathrm{AD}_{\mathrm{h}}=\mathrm{AD}_{\mathrm{r}}=\mathrm{AD}\right)$, and that it is independent of $\delta$ and $t_{\mathrm{d}}$. Secondly, we note that under experimental conditions with limited gradient amplitudes, $\mathrm{RD}_{\mathrm{r}} \approx 0$ for small axon diameters [55]. Equation 1 now provides the MRI signal 
$S$ using six model parameters: $S_{0}, f_{\mathrm{r}}, \theta, \psi, \mathrm{AD}$ and $\mathrm{RD}_{\mathrm{h}}$. For experiments performed with diffusion encoding perpendicular to the nerve $(\mathbf{n} \cdot \mathbf{u}=0)$, the model can be simplified so that it describe the radial signal attenuation curve RS using only three model parameters: $S_{0}, f_{\mathrm{r}}$, and $\mathrm{RD}_{\mathrm{h}}$, i.e. $\mathrm{RS}=S_{0}\left(f_{\mathrm{r}}+\left[1-f_{\mathrm{r}}\right] \exp \left[-b \mathrm{RD}_{\mathrm{h}}\right]\right)$. In isotropic tissue, this model for $R S$ also describes $S$ in any direction.

This highly simplistic model of diffusion in white matter is based on the recognition that it is the organisation of cell membranes around axons that mainly determines the diffusivity in white matter [35]. Features of white matter that are less relevant to the model include, for example, the neurofilaments in the axonal cytoplasm [56]. Internal susceptibility-induced gradients are also negligible [57]. The very minimal model neglects water in glial cells, which is assumed to be either in fast exchange with the extracellular space and thus a part of the hindered fraction [49], or to represent a negligible fraction of the total MR signal. Despite its simplicity, the very minimal model provides valuable insights; for example, it predicts that RD obtained in DTI is sensitive to the axon density according to $[55,58]$

$\mathrm{RD} \approx\left(1-f_{\mathrm{r}}\right) \mathrm{RD}_{\mathrm{h}}$,

when assuming that the axon density correlates with $f_{\mathrm{r}}$. This relation is also valid to describe the mean diffusivity (MD) in isotropic tissue such as many tumours, which has led to the use of MD as a proxy for the cellularity of tumours [59].

The compartment model and the resolution limit

The very minimal model can be expanded to include the axon diameter $d$, by modelling $\mathrm{RD}_{\mathrm{r}}$ as a function of the axon diameter, $d$, and the intra-axonal diffusion coefficient, $D_{\text {intra }}$, as well as the experimental parameters $\delta$ and $t_{\mathrm{d}}$. In the analysis of restricted diffusion, it is informative to define two dimension-less variables $\alpha$ and $\beta$ according to

$\alpha=4 \delta D_{\text {intra }} / d^{2}, \quad \beta=4 \Delta D_{\text {intra }} / d^{2}$.

The value of $\mathrm{RD}_{\mathrm{r}}$ can now be calculated by using the approximation of a Gaussian phase distribution (GPD) [6062], according to

$\operatorname{RD}_{\mathrm{r}}(\alpha, \beta)=k^{2}(\alpha, \beta) d^{2} / 2 t_{\mathrm{d}}$.

For diffusion restricted to a cylinder and with gradients applied perpendicular to the main axis of the cylinder, $k^{2}(\alpha, \beta)$ is given by $[63,64]$

$k^{2}(\alpha, \beta)=\sum_{m=1}^{\infty} \frac{2 \alpha a_{m}-2+2 \mathrm{e}^{-\alpha a_{m}}+\left(2-\mathrm{e}^{\alpha a_{m}}-\mathrm{e}^{-\alpha a_{m}}\right) \mathrm{e}^{-\beta a_{m}}}{\alpha^{2} a_{m}^{3}\left(a_{m}-1\right)}$

where $a_{m}$ is defined by $J^{\prime}\left(a_{m}^{1 / 2}\right)=0$, so that $\left(a_{m}\right)^{1 / 2}$ are the roots of the derivative of the Bessel function of the first kind and order one. Other expressions are available for diffusion restricted by parallel planes or a sphere [64].

The variable $D_{\text {intra }}$ in Eq. 5 is often assumed to be scalar (i.e., isotropic intra-axonal diffusion), with a value equal to $\mathrm{AD}$ or fixed to a value obtained from the literature. This model thereby describes RS using four model parameters: $S_{0}, f_{\mathrm{r}}, \mathrm{RD}_{\mathrm{h}}$, and $d$, and will here on be denoted as the compartment model. A similar model was called the minimal model of white matter diffusion by Zhang et al. [65]. The reason for $D_{\text {intra }}$ not being included as a free model parameter here is that its value is difficult to measure directly, since $\mathrm{RD}_{\mathrm{r}}$ only approaches $D_{\text {intra }}$ when $t_{\mathrm{d}} \rightarrow 0$. However, $\mathrm{RD}_{\mathrm{r}}<<D_{\text {intra }}$ under most experimental conditions when performing diffusion MRI on neural tissue.

There is a lower limit that we call the resolution limit $d_{\text {min }}$, below which the axon diameter is difficult to estimate accurately. The appearance of the resolution limit is evident in $q$-space analysis [31, 32, 66], but it appears also in model-based analysis. Alexander et al. [67] compared the accuracy of axon diameter estimates from acquisition protocols optimised for an animal and a clinical MRI scanner, featuring gradient systems with $g_{\max }=140$ and $60 \mathrm{mT} / \mathrm{m}$, respectively. The study did not explicitly evaluate the value of $d_{\min }$, but it can be approximated from the results presented to 2.5 and $3.5 \mu \mathrm{m}$ for the protocols optimised for the animal and human system, respectively. Nilsson et al. [68] similarly showed that axon diameter estimates are accurate only above $4-5 \mu \mathrm{m}$, based on results from Monte Carlo simulations performed for a protocol designed for a system with $g_{\max }=100 \mathrm{mT} / \mathrm{m}$. The inaccurate axon diameter estimates are caused by the quick approach of $\mathrm{RD}_{\mathrm{r}}$ to zero as $d$ decreases and $\alpha$ increases (Fig. 2). For example, $\mathrm{RD}_{\mathrm{r}} \approx 0.01 \mu \mathrm{m}^{2} / \mathrm{ms}$ for $d=4 \mu \mathrm{m}$, $\delta=10 \mathrm{~ms}$, and $\Delta=20 \mathrm{~ms}$. In $q$-space analysis, the resolution limit is inversely proportional to the maximum

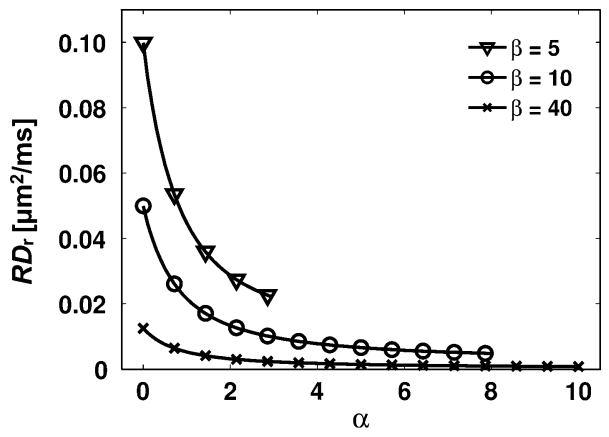

Fig. 2 The value of $\mathrm{RD}_{\mathrm{r}}$ quickly approaches zero as $\alpha$ increase. The graph illustrates Eq. 6 for various combinations of $\alpha$ and $\beta$ in Eq. 5 for $d=4 \mu \mathrm{m}, D_{\text {intra }}=2 \mu \mathrm{m}^{2} / \mathrm{ms}$, and varying values of $\delta$ and $\Delta$, so that $\alpha=1$ corresponds to $\delta=2 \mathrm{~ms}$. The maximum value of $\alpha$ is determined by $\delta_{\max }=\Delta-t_{\mathrm{rf}}$, here with $t_{\mathrm{rf}}=4 \mathrm{~ms}$. In practice, values of $\mathrm{RD}_{\mathrm{r}}$ below approximately $0.02 \mu \mathrm{m}^{2} / \mathrm{ms}$ may be difficult to distinguish from $\mathrm{RD}_{\mathrm{r}}=0$ 
$q$-value [66]. Reducing the resolution limit requires higher values of $g_{\max }$, which permits $q_{\max }$ to increase and $\alpha$ to decrease, which according to Lätt et al. [32] gives $d_{\min } \propto g_{\max }^{-1 / 3}$. For model-based analysis, however, the resolution limit scales according to $d_{\min } \propto g_{\max }^{-1 / 2}$, i.e., modelbased analysis put less strong requirements on scanner hardware than $q$-space analysis does, according to preliminary results by Nilsson and Alexander [69]. In addition to being dependent on $g_{\max }$, the resolution limit also depends on the noise level. For example, Alexander showed that two systems with diameters of 2 and $4 \mu \mathrm{m}$ became inseparable in terms of the estimated values of $d$ when the signal-to-noise ratio (SNR) was reduced from 50 to 20 [19].

The compartment model relies on a few assumptions. First, it assumes that axons are well modelled by impermeable, parallel and equal-sized cylinders. This assumption can be relaxed, as discussed below. Secondly, it assumes that an inaccurate prior value of $D_{\text {intra }}$ does not hamper the accuracy of other model parameters. To our knowledge, this assumption has not been investigated in detail. Thirdly, it assumes that $\mathrm{RD}_{\mathrm{h}}$ is independent of $\delta$ and $t_{\mathrm{d}}$, which is probably an unproblematic assumption. Fourthly, it assumes that the GPD approximation describes $\mathrm{RS}_{\mathrm{r}}$ sufficiently well. This assumption is valid for most experimental conditions [70], but not for $\alpha<<1$ and $\beta>>1$, since the signal curve then takes the shape of a diffraction pattern [71-73]. The amplitude of the highest diffraction peak is, however, less than $5 \%$ of $S_{0}$, although it may increase, for example, in the presence of a surface relaxation sink which enhance the relaxation rate close to the membrane [74]. Nevertheless, the GPD approximation is generally valid until less than $10 \%$ of $S_{0}$ remains, as shown both by simulations and experiments [68, 75]. Another condition that invalidates the GPD approximation is when $\beta<<1$. This condition may result in apparently biexponential signal-versus- $b$ curves from a single compartment [76]. In the context of diffusion MRI using clinical MRI scanners, however, this condition is of little concern for most protocols since $\beta<<1$ only for $d$ greater than $20 \mu \mathrm{m}$.

Modelling of axon diameter distributions

Nerves are typically composed of axons of varying diameters, which can be incorporated in the model of $R S_{\mathrm{r}}$ according to

$\mathrm{RS}_{\mathrm{r}}=\int \rho\left(d^{\prime} \mid d, \sigma_{\mathrm{d}}\right) \exp \left(-b \mathrm{RD}_{\mathrm{r}}\left(d^{\prime} \mid \delta, t_{\mathrm{d}}\right)\right) \mathrm{d} d^{\prime}$,

where $\rho\left(d^{\prime} \mid d, \sigma_{\mathrm{d}}\right)$ is the volume-weighted axon diameter distribution with mean $d$ and standard deviation $\sigma_{\mathrm{d}}$. This model will be referred to as the diameter distribution model, but it has also been described as the AxCaliber model [21]. In that model, the axon diameter distribution is modelled by a gamma distribution, with shape and scale parameters given by $\left(d / \sigma_{\mathrm{d}}\right)^{2}$ and $\sigma_{\mathrm{d}}^{2} / d$.

In the presence of a distribution of axon diameters, $\mathrm{RS}_{\mathrm{r}}$ becomes apparently biexponential [77]. However, $\mathrm{RS}_{\mathrm{r}}$ is approximately monoexponential under the experimental limitations imposed by the clinical MRI scanner, even for relatively large values of $\sigma_{\mathrm{d}}$ (Fig. 3). In the analysis of high $b$-value data acquired in vivo, Zhang et al. [65] showed in a conference abstract that a model assuming equal-sized axons produces higher estimates of the average axon diameter than a model assuming a diameter distribution. The source of this bias may be partly explained by Fig. 3, which shows that the slope of the signal-versus- $b$ curve increases as $\sigma_{\mathrm{d}}$ increases, even as the average diameter is fixed. The estimated value of $f_{\mathrm{r}}$ is less influenced than the axon diameter by whether a single compartment size is assumed or a size distribution is incorporated in the model [78].

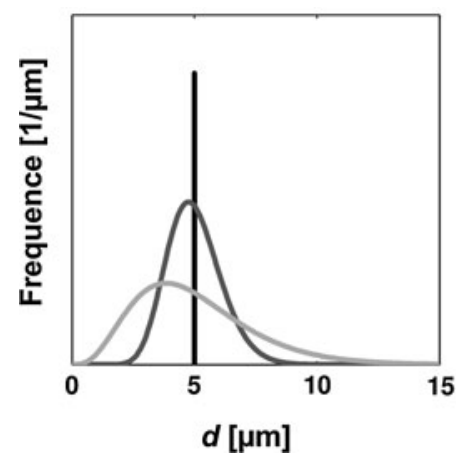

Fig. 3 Left: Three gamma distributions of axon diameters, all with average diameters of $5 \mu \mathrm{m}$, but with $\sigma_{\mathrm{d}}=0,1.1$ and $2.4 \mu \mathrm{m}$. Middle: corresponding $\mathrm{RS}_{\mathrm{r}}$-versus- $b$ curves from the three distributions, matched in grey scale with the distribution panel. Right: Values of $\mathrm{RD}_{\mathrm{r}}$ used to generate the signal curves, versus $d$. The values were
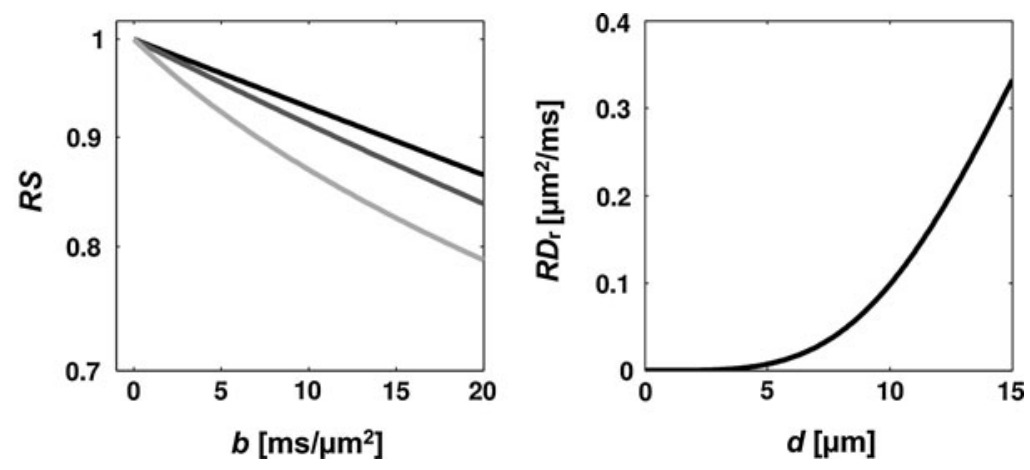

calculated from Eqs. 6 and 7, assuming a typical diffusion MRI protocol with $\delta=20 \mathrm{~ms}$ and $t_{\mathrm{d}}=18 \mathrm{~ms}$. Note that all curves are approximately monoexponential up to the maximum $b$-value achievable with clinical MRI scanners $\left(b_{\max } \approx 5 \mathrm{~ms} / \mu \mathrm{m}^{2}\right.$ for the given values of $\delta$ and $t_{\mathrm{d}}$, with $g_{\max }=100 \mathrm{mT} / \mathrm{m}$ ) 
Orientation dispersion and axonal undulation

Axons are normally modelled as being parallel, but this assumption may be invalid. Leergard et al. [79] obtained axonal orientation distributions by manually recording individual fibre orientations on myelin-stained histological sections. The full-width at half-maximum (FWHM) of the angular orientation distribution was $34^{\circ}$ in the densely packed corpus callosum. Axonal undulation also induces axonal orientation dispersion [80].

Axonal orientation dispersion can be incorporated into the model of RS as described by Zhang et al. [81], but is here adapted to the form of Eq. 8, according to

$$
\begin{aligned}
\mathrm{RS}_{\mathrm{r}}= & \int \rho(\mathbf{v} \mid \mathbf{u}, \kappa) \\
& \exp \left(-\mathrm{b}(\mathbf{n} \cdot \mathbf{v})^{2} \mathrm{RD}_{\mathrm{r}}-\mathrm{b}\left(1-(\mathbf{n} \cdot \mathbf{v})^{2}\right) \mathrm{AD}_{\mathrm{r}}\right) \mathrm{d} \mathbf{v},
\end{aligned}
$$

where $\rho(\mathbf{v} \mid \mathbf{u}, \kappa)$ is the orientation distribution around the direction $\mathbf{u}$ with a dispersion factor $\kappa$. This model will be called the orientation dispersion model, described using five model parameters: $S_{0}, f_{\mathrm{r}}, \mathrm{RD}_{\mathrm{h}}, d$, and $\kappa$, assuming $\mathrm{AD}_{\mathrm{r}}$ is fixed to some prior value.

The effect on $\mathrm{RS}_{\mathrm{r}}$ of an orientation dispersion has been investigated experimentally and in simulations by Avram et al. [72]. The results showed that a wider orientation distribution led to faster signal attenuation at low $b$-values and less signal remaining at high $q$-values. Analysing such data with the compartment model would presumably result in higher values of $\mathrm{RD}_{\mathrm{h}}$ and lower values of $f_{\mathrm{r}}$.

Zhang et al. [81] fitted the compartment model, which assumes parallel axons, to data simulated from the orientation dispersion model. This resulted in over- and underestimated values of $d$ and $f_{\mathrm{r}}$, respectively, although these biases were almost recovered by instead fitting the orientation dispersion model to the simulated data. Effects of the resolution limit, however, prevented accurate estimation of $d$ below approximately $4 \mu \mathrm{m}$. Drawing on the weak signal dependency for small axon diameters, Zhang et al. [82] refined the model to assume $d=0 \mu \mathrm{m}$, which allowed for improved estimation of $\kappa$. The resulting model, called neurite orientation dispersion and density imaging (NODDI), allows the orientation dispersion to be estimated in the human brain from data obtained in as little as $10 \mathrm{~min}$.

Axons in extracranial white matter and in the optic nerve undulate, i.e., they follow approximately sinusoidal paths [83]. For the optic nerve, the non-straightness is easily appreciated from reconstructed 3D segments of axons (Fig. 4). Diffusion measurements performed in sinusoidally undulating axons yields results similar to those performed in the presence of orientation dispersion according to Monte Carlo simulations by Nilsson et al. [80], although there is a fundamental difference between orientation dispersion at the micro- and macroscopic levels. In axons that undulate with wavelengths of a few tens of microns, $d$ is overestimated by an amount proportional to the undulation amplitude. This bias is probably not recoverable by improved modelling, since the water molecules have time to sample one or more complete undulations during the diffusion time, so that the effective restriction length is actually larger than the axon diameter. A similar argument can be applied for axons that vary in diameter, for example, those in the optical nerve which varies up to a factor of two in diameter over a distance of $12 \mu \mathrm{m}$ [45]. In the case of undulation wavelengths of a hundred microns or more, the differently oriented segments of the axon may be regarded as non-exchanging [80], thereby meeting the assumptions in the orientation dispersion model. Stretching a nerve with undulating axons reduces the undulation amplitude, which would result in less of an overestimation of the axon diameter, and probably also in alterations of the diffusion characteristics measured by DTI [80].

The two-compartment exchange model and membrane permeability

In the presence of exchange between two water components, the diffusion-weighted signal can be predicted by the Kärger equations [84, 85]. These equations are derived from the Bloch-Torrey equations [86], where the magnetisation $\mathbf{S}$ in the water components are related by rate equations $\mathrm{d} \mathbf{S} / \mathrm{d} t=\mathbf{A} \cdot \mathbf{S}$. The mixing matrix is given by $\mathbf{A}=-(2 \pi q)^{2} \mathbf{D}+\mathbf{K}$, so that the solution to the rate equations provides an expression for the total signal $S$ according to [78]

$S\left(q, t_{\mathrm{d}}\right)=S_{0} \mathbf{1}^{\mathrm{T}} \exp \left(-(2 \pi q)^{2} t_{\mathrm{d}} \mathbf{D}+t_{\mathrm{d}} \mathbf{K}\right) \cdot \mathbf{f}$,

where $S_{0}$ is the signal acquired without diffusion weighting, $\mathbf{1}$ is a column vector of ones. For the twocomponent system discussed previously, $\mathbf{D}=\operatorname{diag}\left(D_{\mathrm{h}}, D_{\mathrm{r}}\right)$, i.e., the model assumes that the GPD approximation is valid in all compartments. Moreover, $\mathbf{f}=\left[f_{\mathrm{h}}, \mathrm{f}_{\mathrm{r}}\right]$, and the exchange matrix $\mathbf{K}$ is given by

$\mathbf{K}=\left[\begin{array}{ll}-k_{\mathrm{h}, \mathrm{r}} & +k_{\mathrm{r}, \mathrm{h}} \\ +k_{\mathrm{h}, \mathrm{r}} & -k_{\mathrm{r}, \mathrm{h}}\end{array}\right]$,

where conservation of mass gives $f_{\mathrm{h}} k_{\mathrm{h}, \mathrm{r}}=f_{\mathrm{r}} k_{\mathrm{r}, \mathrm{h}}$ under the assumption that $f_{\mathrm{i}}$ represents the total mass of component $i$. For experiments performed using a double PGSE sequence instead of the conventional single PGSE sequence, the twocompartment model in Eq. 10 can be simplified to only include four model parameters in the so-called filtered 
exchange imaging (FEXI) experiment [87]. FEXI gives the apparent exchange rate (AXR), which is related to the exchange rate according to $\mathrm{AXR}=\left(k_{\mathrm{r}, \mathrm{h}} \cdot f_{\mathrm{h}}\right)^{-1}$. Details regarding that experiment are, however, outside the scope of this review.

For cells embedded in a homogeneous medium the outward exchange rate from the cells is given by

$K_{\mathrm{r}, \mathrm{h}}=P_{\mathrm{d}}(A / V)_{\mathrm{r}}=1 / \tau_{i}$,

where $P_{\mathrm{d}}$ is the diffusional membrane water permeability, $(A / V)_{\mathrm{r}}$ is the surface-to-volume ratio of the cell, and $\tau_{\mathrm{i}}$ is the mean residence time for a molecule in the cell, or the intracellular exchange time [13]. The diffusional water membrane permeability $P_{\mathrm{d}}$ is affected by the properties of the lipids in the membrane and by water-channel proteins embedded in the membrane $[88,89]$. It generally increases smoothly with the temperature, although it may increase sharply at certain temperatures $[13,88]$. Note the difference between the osmotic and diffusional permeability, where the former is generally larger than the latter and refers to the permeability measured in the presence of an osmotic pressure gradient over the membrane [90]. The
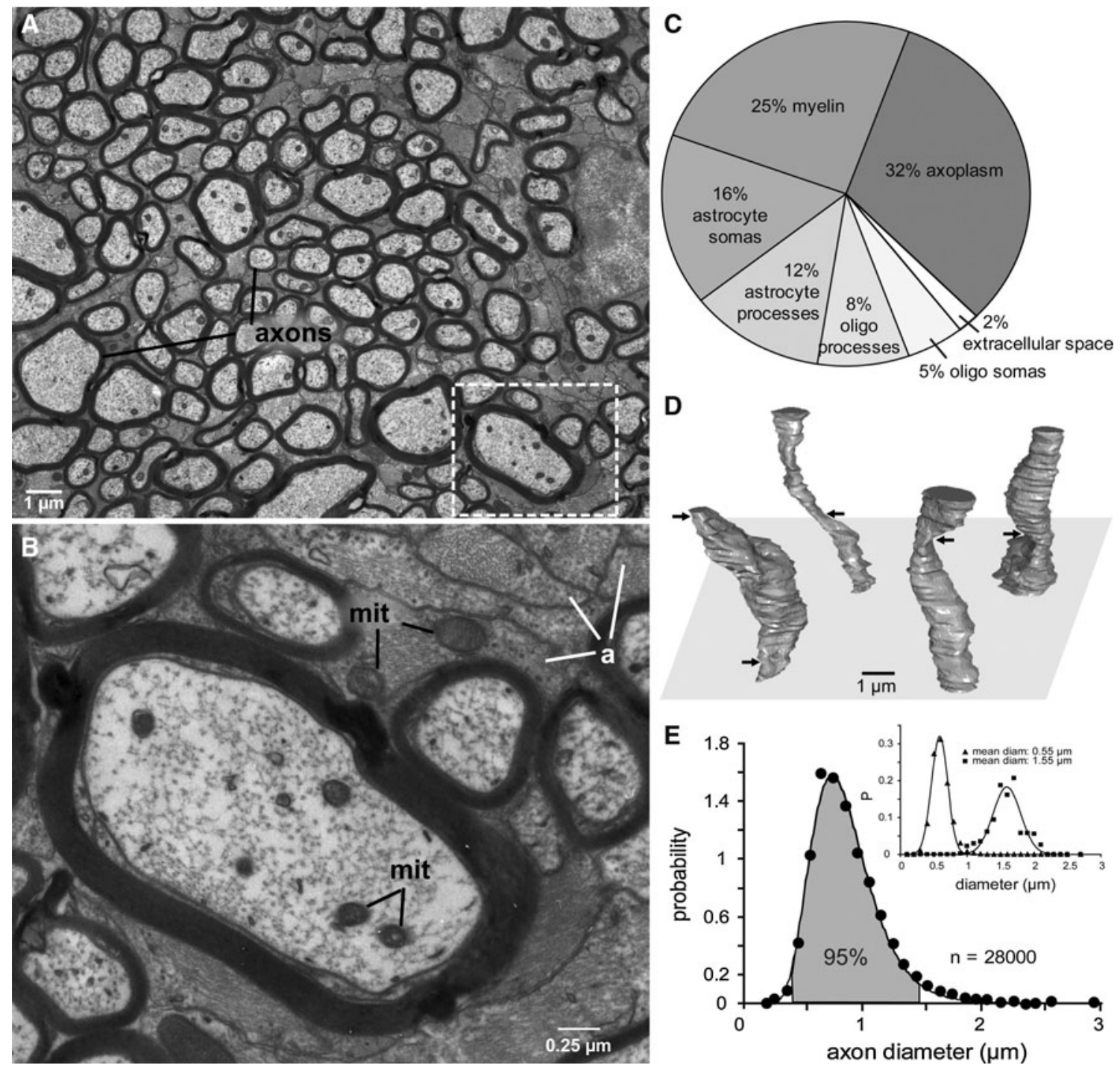

Fig. 4 a Myelinated axons in the optic nerve vary in diameter by tenfold and are separated from each other by astrocyte processes (electron micrograph). b Higher magnification of the boxed region in A shows mitochondria (mit) in axons and astrocyte processes (a). c Allocation of space in the optic nerve. d Optic axons reconstructed from inner diameters over a length scale of $12 \mu \mathrm{m}$. The axons vary markedly in caliber $(\mathrm{max} / \mathrm{min}=2.0 \pm 0.6 \mu \mathrm{m} ; n=1,200)$. Arrows mark constrictions. None of these constrictions were nodes of Ranvier. e Distribution of diameters is skewed with thin axons predominating. Solid line is a lognormal fit. Inset: Distribution of diameters along the reconstructed segments for a subset of axons with mean diameter $0.55 \mu \mathrm{m}(n=1,100)$ and $1.55 \mu \mathrm{m}(n=500)$. Solid lines are Gaussian fits. Reproduced from Perge et al. [45] with permission from Journal of Neuroscience 
diffusion NMR/MRI experiment measure the permeability under steady-state conditions, and thereby yields the diffusional permeability [91].

The model in Eq. 10 is here on called the two-compartment exchange model, and it describes RS using five parameters: $S_{0}, f_{\mathrm{r}}, \mathrm{RD}_{\mathrm{h}}, d$, and $\tau_{\mathrm{i}}$. Special cases of this model allow $\tau_{\mathrm{i}}$ to be inferred from constant-gradient experiments, in which $g$ is fixed while $t_{\mathrm{d}}$ is varied $[92,93]$. This approach provides accurate estimates of $\tau_{\mathrm{i}}$, but for long diffusion times and values of $g_{\max }$ above those normally available with clinical MRI scanners. Instead of the approach used in constant-gradient experiments of only collecting limited data, a large set of experimental conditions with varying values of $\delta, t_{\mathrm{d}}$ and $b$ can be acquired. This allows the full two-compartment exchange model to be fitted to the data.

Although the two-compartment exchange model is derived based on an assumption incompatible with the notion of restricted diffusion; that both components show Gaussian diffusion where the mean-squared distances increase linearly with time, it predicts the outcome of a single PGSE experiment well in most cases $[68,86]$. For example, Nilsson et al. [23] evaluated the performance of the model using Monte Carlo simulations, for a protocol with $\delta=50 \mathrm{~ms}, t_{\mathrm{d}}=64-256 \mathrm{~ms}$, and $b_{\max }=28 \mathrm{~ms} / \mu \mathrm{m}^{2}$. The results showed that effects of both restricted diffusion and exchange can be observed for some microstructural configurations in signal-versus- $b$ curves obtained using a clinical scanner (Fig. 5). Another study performed a similar evaluation using a protocol with $\delta=30 \mathrm{~ms}$, $t_{\mathrm{d}}=30-60 \mathrm{~ms}$, and $b_{\max }=20 \mathrm{~ms} / \mu \mathrm{m}^{2}$ [68]. These two studies showed that the two-compartment model generally provides accurate estimates of the values that were used in the simulation, except for $d$ below the resolution limit. In addition, two other exceptions were found. First, the exchange time was accurately estimated only when being on the same order of magnitude as the maximal diffusion time employed in the measurements. For example, Nilsson et al. [68] showed that $\tau_{\mathrm{i}}$ was accurately estimated for $\tau_{\mathrm{i}}<300 \mathrm{~ms}$, compared to the maximal diffusion time of $t_{\mathrm{d}}=60 \mathrm{~ms}$. Second, fast exchange demand high $q$ values
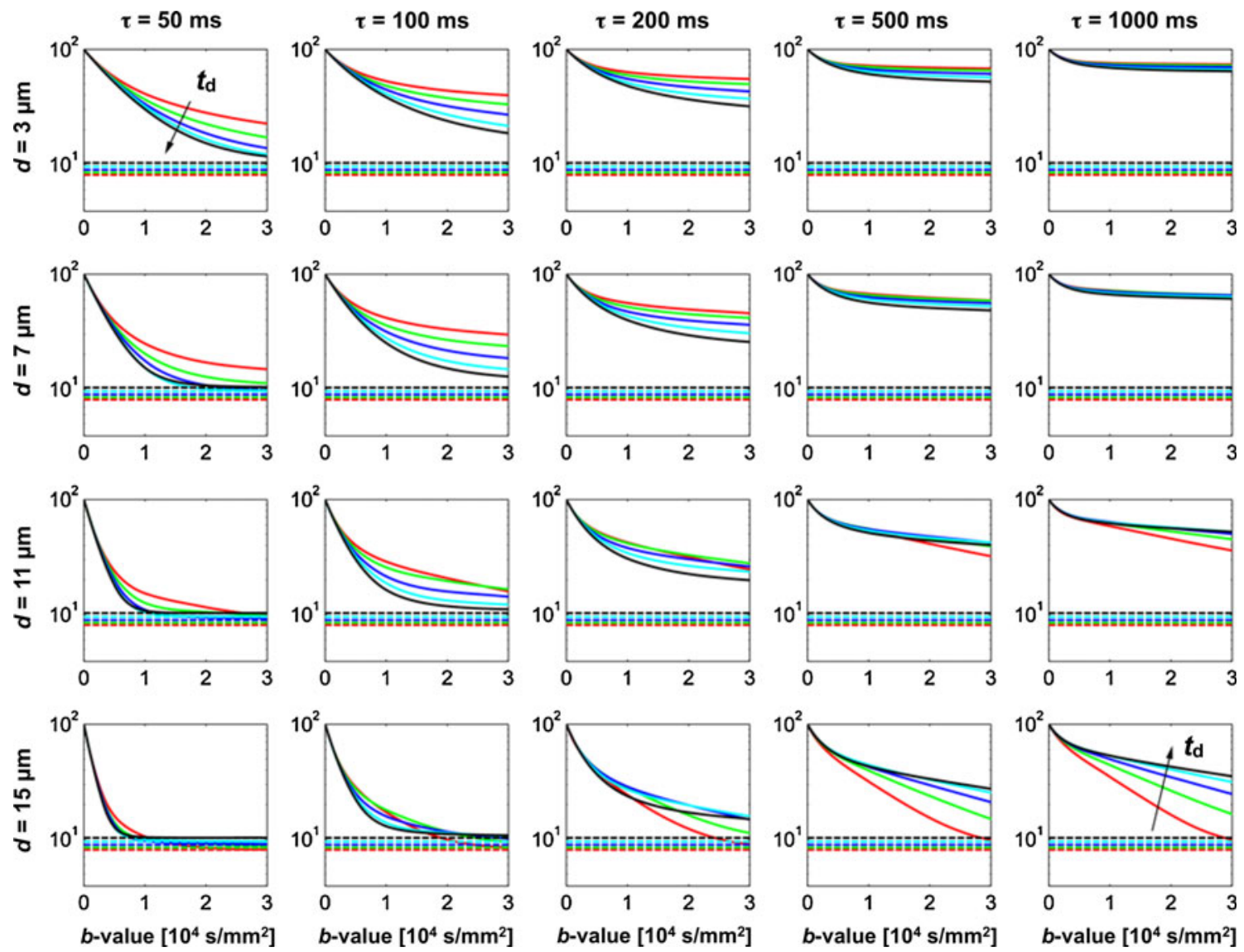

Fig. 5 Signal curves simulated with $\delta=50 \mathrm{~ms}$ and diffusion times from 64 to $256 \mathrm{~ms}$, shown in red to black, order according to the arrows. Columns show varying exchange times, while rows show varying diameters. Note that the amplitude of the signal-versus$b$ curves increase at high $b$-values for prolonged $t_{\mathrm{d}}$ when effects of restricted diffusion dominate (lower right), while the opposite occurs when effects of exchange dominate (upper left). Dashed lines represent the magnified noise floor. Note the unit of $b$, where $10^{4} \mathrm{~s} / \mathrm{mm}^{2}=10^{6} \mathrm{~s} / \mathrm{cm}^{2}=10 \mathrm{~ms} / \mu \mathrm{m}^{2}$. Reproduced from Nilsson et al. [23] with permission from Elsevier 
in order to be observable according to the "shutter speed" analysis of Lee and Springer [94], and is accurately quantified only if the exchange is barrier limited [86].

The concept of barrier limited exchange relates to an assumption in the Kärger equations; that the exchanging components are well mixed so that all particles have equal probabilities of switching components during $\tau_{\mathrm{i}}$. This assumption is valid in compartmentalised systems only when $\tau_{\mathrm{i}}>d^{2} / 2 D_{\mathrm{i}}$, i.e., barrier limited exchange as discussed by Fieremans et al. [86]. Violation of this condition leads to inaccurate parameter estimates. For example, Nilsson et al. [23] showed that the estimated values of $\tau_{\mathrm{i}}$ and $f_{\mathrm{r}}$ became inaccurate for $d>8 \mu \mathrm{m}$. Another study by Nilsson et al. [68] similarly found that $f_{\mathrm{r}}$ was underestimated for large values of $d$ and low values of $\tau_{\mathrm{i}}$, but showed that this problem can be partly mitigated by matching acquired data with data obtained from Monte Carlo simulations that have been performed with varying model parameters and stored in a database. Other studies have also encountered the concept of barrier-limited exchange, but discussed it in other terms [78, 95, 96]. The membrane permeability at which the exchange is no more barrier limited also represents the point at which increased permeability results in increased ADC values, as shown in Fig. 6 [97].

Under conditions in which the exchange is not barrierlimited, but rather limited by the time necessary to diffuse across the cell, the exchange time in a cylinder is given by [93]

$\tau_{\mathrm{i}}=d^{2} / 32 D_{\text {intra }}+d / 4 P_{\mathrm{d}}$,

rather than by Eq. 12, with $(V / A)_{\mathrm{i}}=d / 4$ for a cylinder.

\section{Summary of models}

Table 1 shows a summary of the models describing RS in white matter, although these models could equally well be employed to describe $S$ independently of the diffusion encoding direction in isotropic systems. Expanding the models is generally straightforward as for example the inclusion an isotropic CSF component [67]. The models could also be combined, for example, to model exchange, a diameter distribution, and orientation dispersion, using seven model parameters to describe RS. Accurate representation of the white matter microstructure probably requires all these features to be present in the model. In addition, two or three hindered and restricted components with different orientations are required to model the diffusion in white matter regions that contain multiple fibre populations with different orientations. Behrens et al. [98] suggested that at least a third of all white matter voxels contain more than one fibre population. Potentially, nearly all white matter voxels may contain crossing fibres [99].
Model selection is not a trivial matter, because clearly the microstructure of the white matter is highly complex in most if not all parts of the brain. Estimating all properties of all fibre populations may not even be possible, so simplifications are required. The NODDI model by Zhang et al. [82] is a good example of where simplifications allows more precise estimates of relevant parameters, but model simplification requires approximations that may be invalid. For example, the assumption of non-exchanging compartments is invalid in sub-acute ischemic stroke lesions [100]. However, the use of more complex models, having a greater number of model parameters, is not always feasible, since fitting the model parameters may capture features of the signal noise rather than underlying microstructure. To avoid overfitting, testing whether the data support a complex model over a simple model can be done with an $F$ test, for example, as performed by Kiselev and Il'yasov [101]. They showed that the kurtosis

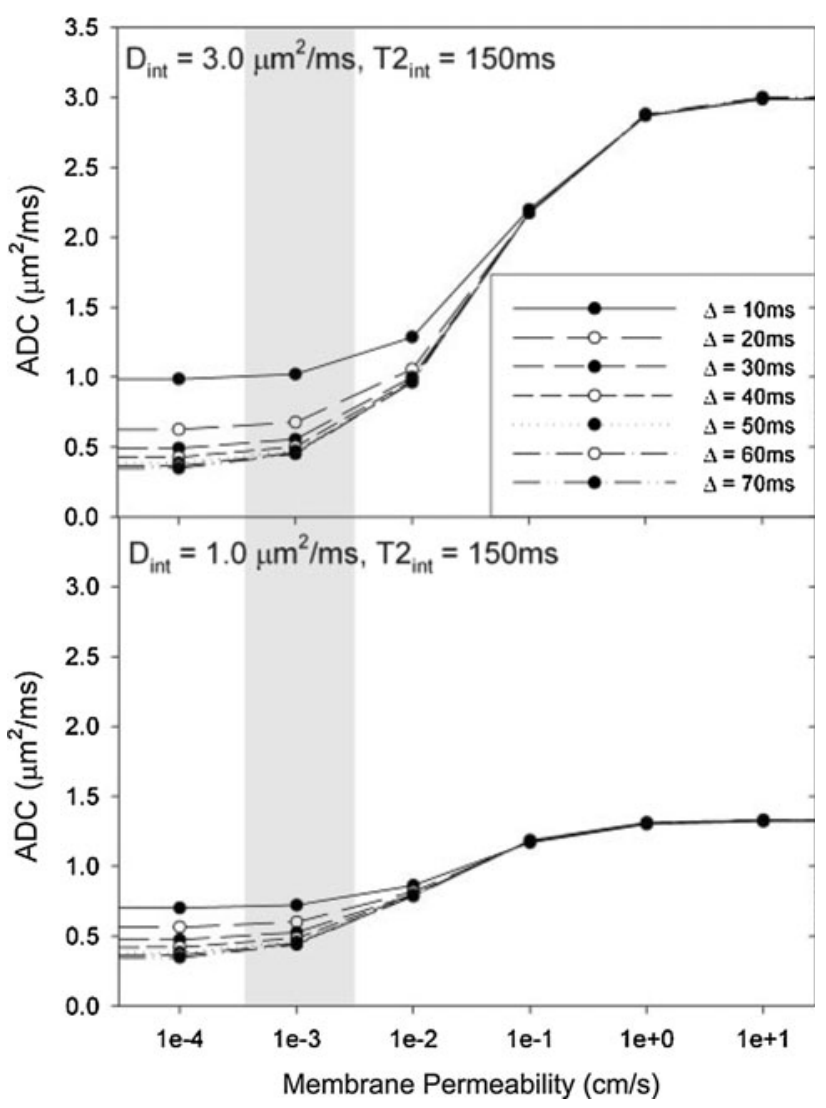

Fig. 6 Variations in cell membrane permeability impact the ADC strongly for very high permeability values only. Calculated ADC values of water were plotted against the membrane permeability, with lines connecting simulations with identical diffusion times. Top and bottom panels depict simulation results with a combination of $D_{\text {intra }}=1.0$ and $3.0 \mu \mathrm{m}^{2} / \mathrm{ms}$, respectively, with equal relaxivities in the two compartments of $150 \mathrm{~ms}$. Shaded regions highlight physiologically relevant membrane permeability values in healthy cells. Reproduced from Harkins et al. [97], with permission from John Wiley and Sons 
Table 1 Summary of models describing RS in white matter, where $n$ denotes the number of model parameters

\begin{tabular}{llll}
\hline Model name & Model parameters & $n$ & Comments \\
\hline Very minimal & $S_{0}, f_{\mathrm{r}}, \mathrm{RD}_{\mathrm{h}}$ & 3 & Assumes $\mathrm{RD}_{\mathrm{r}}=0$ \\
Compartment & Minimal $+d$ & 4 & Similar to the CHARMED model [20] \\
Diameter distribution & Minimal $+d, \sigma_{\mathrm{d}}$ & 5 & Similar to the AxCaliber model [21] \\
Two-compartment exchange & Compartment $+\tau_{\mathrm{i}}$ & 5 & Based on the Kärger equations \\
Orientation dispersion & Compartment $+\kappa$ & 5 & Model by Zhang et al., also requires AD, which can be assumed to be equal to $D_{\mathrm{i}}$ \\
Undulation & Minimal $+A, L, \mathrm{AD}$ & 6 & RS given by a propagator model [80] \\
FEXI & $\mathrm{ADC}, \sigma, \mathrm{AXR}$ & 3 & In addition to these three model parameters, $S_{0}$ is included for each mixing time \\
DKI & $S_{0}, \mathrm{RD}, \mathrm{RK}$ & 3 & The full diffusional kurtosis model uses 22 model parameters [26] \\
Biexponential & $S_{0}, f_{\mathrm{s}}, D_{\mathrm{f}}, D_{\mathrm{s}}$ & 4 & Modelling a fast and a slow diffusion tensor requires 14 model parameters \\
\hline
\end{tabular}

All models could be extended to describe the signal in any direction by using three more model parameters that define the direction of the fibre $(\theta, \varphi)$ and the axial diffusivity (AD). The FEXI, diffusional kurtosis, and biexponential models are included for comparison

model (three parameters) could be used just as well as the biexponential model (four parameters) to fit data acquired in vivo with high $b$-values in $20-41 \%$ of the grey matter voxels investigated. This means that not all of the data acquired supported the biexponential model. The Bayesian information criterion can also be used to compare models. Using data acquired in the corpus callosum of perfusionfixated rat brains, Panagiotaki et al. [102] evaluated 47 analytic models of diffusion in multiple non-exchanging compartments with up to 11 model parameters. They found that models incorporating an intra-axonal component having restricted diffusion generally explained the data better than models assuming hindered diffusion in all components. However, diffusion MRI data alone may be insufficient to select between models of equal complexity. For models having an equal number of model parameters, these may be transformed from one model to the other. For example, the number of model parameters in the very minimal model (three) is equal to that of the kurtosis model (for measurements performed in a single direction). Consequently, the parameters in the two models can be related according to $f_{\mathrm{r}}=\mathrm{RK} /(\mathrm{RK}+3)$ and $\mathrm{RD}=f_{\mathrm{h}} \cdot \mathrm{RD}_{\mathrm{h}}$, where $\mathrm{RK}$ is the radial kurtosis [30]. Three of the models in Table 1, the diameter distribution model, the two-compartment exchange model, and the orientation dispersion model, all describe the signal curves using five model parameters. Finding the optimal model in such a case requires careful model evaluation [102]. Choosing the optimal model could also be aided by the contribution of independent external information, for example, that acquired by microscopy. The FEXI protocol could also contribute with independent information regarding exchange, since it is sensitive specifically to the exchange between the slow and fast diffusion components [87, 103].

\section{Extracellular diffusion}

In addition to the concepts included in the models above, the structure of the extracellular space will also influence the water diffusion. The extracellular space is tortuous, which in nerves results in diffusion that is more hindered in the direction perpendicular to the nerve than parallel to it, according to

$\mathrm{RD}_{\mathrm{h}}=\mathrm{AD}_{\mathrm{h}} / \lambda^{2}$,

where $\lambda$ is the tortuosity factor. For ion diffusion in the rat cerebellum, this factor has been measured as $\lambda=1.55 \pm 0.05$ [104], however, the value of $\lambda$ depends on the fractional volume of the extracellular space $\left(v_{\text {extra }}\right)$. For example, Lipinski et al. [105] reported that $\lambda=v_{\text {extra }}^{-0.41}$, based on particle simulations on digitised images of histological sections. Other relations have also been employed, for example $\lambda^{2}=1+\left(1-v_{\text {extra }}\right)^{3 / 2}$ by Hall et al. [106] and $\lambda^{2}=v_{\text {extra }}^{-1}$ by Alexander [67].

By using a model that relates $\lambda$ and $v_{\text {extra }}$, the number of model parameters may in some cases be reduced by one, since Eq. 14 relates $\mathrm{RD}_{\mathrm{h}}$ to $A D_{\mathrm{h}}$. However, the relation between $\lambda$ and $v_{\text {extra }}$ is uncertain and is likely to be influenced also by factors other than $v_{\text {extra }}$, such as the narrow spaces between cells [107]. In addition, the hindered fraction $f_{\mathrm{h}}$ may be an inaccurate proxy of $v_{\text {extra, }}$, since it may represent water from both the extracellular space and from cells in fast exchange with it [49]. Equation 14 may thus be more suitable for post-hoc analysis of estimated model parameters than for incorporation in biophysical models.

\section{Model fitting}

The diffusion MRI experiment is relatively simple to describe from a theoretical point of view, but implementing it and analysing the results is more complicated in practice, as described thoroughly elsewhere [108, 109]. The most important aspect to consider in the context of biophysical modelling of white matter diffusion is the statistical distribution of the MRI signal. For single-receiver systems, the magnitude signal is Rice-distributed [110, 111]. This distribution is approximately Gaussian if the SNR, defined 
by $\mathrm{SNR}=\mathrm{S} / \sigma$ with $\sigma$ being the standard deviation of the signal in the real or imaginary channel, is higher than approximately two, but has an expectation value of $\sigma(\pi / 2)^{1 / 2}$ when the true signal is zero. This signal bias is known as the rectified noise floor. If $\sigma$ is known, the Rice distribution can be taken into account in the model fitting as shown, for example, by Veraart et al. [112] for the kurtosis model. Multiple receive coils and parallel imaging, techniques widely used today, results in an approximately non-central chi distributed rather than a Rice distributed signal [113115]. The noise level is also non-uniform across the image volume when multiple receive coils are used [116]. Postprocessing such as motion correction also affect the signal distribution [115]. The noise floor bias, which is present also when multiple coils and parallel imaging is used [113, 114], can make it challenging to distinguishing a water signal from environments with highly restricted diffusion $\left(D_{\mathrm{r}} \approx 0\right)$ from the level of the noise floor. Knowledge of the level of the noise floor is thus important in the model fitting.

\section{Model validation in cell suspensions and excised tissue}

Model development has been accompanied by validation experiments in suspensions of, for example, red blood cells and yeast cells. Before comparing in vivo and in vitro results, however, differences in water temperatures could be important to consider since $D_{\text {bulk }}$ and presumably also $D_{\text {intra }}$ increase by approximately $50 \%$ when the temperature increases from 20 to $37{ }^{\circ} \mathrm{C}$ [117]. Measurements at low temperatures are thus beneficial in terms of the resolution limit: in order to keep $D_{\mathrm{r}}, \alpha$, and $\beta$ equal at the two temperatures, the values of $\delta$ and $t_{\mathrm{d}}$ at $37{ }^{\circ} \mathrm{C}$ should be two thirds of those at $20^{\circ} \mathrm{C}$ (Eq. 5). In order to preserve $b_{\max }$, the value of $g_{\max }$ would then need to be approximately $80 \%$ greater at the higher temperature.

Diffusion experiments on excised tissue provide an opportunity to compare model-based estimates of structural parameters of the tissue with independent histology-based estimates. For conclusions drawn from results obtained in excised tissue, the time interval between death and tissue fixation should be considered since it influences diffusion in neural tissue. For instance, the MD in the corpus callosum in a dead brain is reduced from approximately 0.17 to $0.06 \mu \mathrm{m}^{2} / \mathrm{ms}$ during two weeks of brain decomposition [118]. Studies of human tissue are particularly sensitive to this issue, in contrast to animal tissues that may be fixed directly postmortem, or premortem by perfusion fixation. Fixation itself also affects the diffusion; for example, it reduces MD but not FA [119, 120]. Moreover, differences in diffusivity between infarcted and healthy tissue are lost during fixation [119]. The storage time of the fixed tissue only has a minor influence on the MD and FA [121].
Studying exchange using red blood cells and yeast cells suspensions

The exchange rate in red blood cells has been determined using various independent methods such as diffusion NMR and the Kärger model $[117,122,123]$, the $\mathrm{Mn}^{2+}$ doping ${ }^{1} \mathrm{H}$ NMR method [124], and studies of diffusion using internal magnetic field inhomogeneity [125]. The different methods have provided similar results. The diffusional membrane permeability of the mammalian red blood cell is high, with $P_{\mathrm{d}}$ in the range $49-112 \mu \mathrm{m} / \mathrm{s}$ at $37{ }^{\circ} \mathrm{C}$, as measured in various species [124]. The high values of $P_{\mathrm{d}}$ in combination with the small sizes of red blood cells lead to values of $\tau_{\mathrm{i}}$ in the order of 5-10 ms according to Eq. 12, assuming $V / A \approx 0.5 \mu \mathrm{m}[91]$.

The two-compartment exchange model has been used to quantify $\tau_{\mathrm{i}}$ in erythrocyte ghost models. As expected, blocking of the aquaporin channels results in increased values of $\tau_{\mathrm{i}}$ [123]. It has also been shown that the value of $f_{\mathrm{r}}$ estimated from diffusion data is lower than that obtained with an independent method [117]. This underestimation might be expected, since the exchange is not barrier-limited for the high membrane permeability found in red blood cells.

Yeast cells provide a relatively simple model system for diffusion NMR and MRI investigations, in which the exchange rate is much slower than in red blood cells. Åslund et al. [13] used the double PGSE sequence to map the exchange rate in yeast cells, and showed that $P_{\mathrm{d}}$ is dependent on the temperature. Suspensions of yeast cells were used to validate the FEXI model and to compare results obtained with NMR spectrometers and those obtained using a clinical MRI scanner [87]. The results from both platforms resembled each other and agreed with expectations from other studies.

\section{Intracellular diffusion}

Independent estimates of $D_{\text {intra }}$ are valuable in the construction and application of biophysical models of diffusion in tissue. Zhao et al. [126] performed measurements with very short diffusion times and reported $D_{\text {intra }}=2.0 \pm 0.3 \mu \mathrm{m}^{2} / \mathrm{ms}$ in HeLa cells with diameters of approximately $20 \mu \mathrm{m}$, compared to $D_{\text {bulk }} \sim 3 \mu \mathrm{m}^{2} / \mathrm{ms}$ for free water at $37^{\circ} \mathrm{C}$. In another study, Beaulieu and Allen measured the intra-axonal diffusion coefficient in giant axons of the squid, which are large enough $(200-1,000 \mu \mathrm{m})$ to allow for measurements of intra-axonal diffusion coefficients unaffected by restriction effects of the membranes (i.e. $\alpha \rightarrow 0$ and $\beta \rightarrow 0$ in Eq. 7). The values measured were $\mathrm{AD}_{\text {intra }}=1.61 \pm 0.06 \mu \mathrm{m}^{2} / \mathrm{ms}$ and $\mathrm{RD}_{\text {intra }}=1.33 \pm$ $0.09 \mu \mathrm{m}^{2} / \mathrm{ms}$, respectively, which can be compared to $D_{\text {buk }}=2.08 \pm 0.04 \mu \mathrm{m}^{2} / \mathrm{ms}$ for free water at $20{ }^{\circ} \mathrm{C}$ [56] 
Longitudinally ordered neurofilaments within the axons were suggested as the cause of the small anisotropy, i.e., the difference in axial and radial diffusivity of the intra-axonal water. Anisotropy of the intra-axonal diffusivity would likely have some impact on axon diameter estimated obtained by analyses performed with the two-compartment model on data obtained with clinical MRI scanners, since the value of $\mathrm{RD}_{\text {intra }}$ influence $\alpha$ and $\beta$. However, the impact would likely be limited. In both of these studies, $D_{\text {intra }} \sim 2 / 3$ $D_{\text {bulk }}$, but it is even lower in yeast cells [127].

The diffusivity within cells might be inhomogeneous. Sehy et al. [128] showed ADC values in the Xenopus oocyte ranging from $0.5 \mu \mathrm{m}^{2} / \mathrm{ms}$ in the vegetal pole to $1.7 \mu \mathrm{m}^{2} / \mathrm{ms}$ in the nucleus. In neural tissue where the cells are up to three orders of magnitude smaller than the millimetre sized oocyte, such an inhomogeneity probably contributes less to the value of $D_{\mathrm{r}}$ than the size of the cell. Galons et al. [129] investigated rat glioma cells and reported that 50-60\% of the intracellular water has slow diffusion, which also showed evidence of being restricted. This could potentially confound results of model-based analysis that assume a homogeneous intracellular environment, and requires further investigation.

\section{Excised nerves}

Investigations of diffusion in excised tissue using the single PGSE sequence have been performed in several studies, the first of them in 1970s [130, 131]. Most studies have investigated optic and sciatic nerves, spinal cord, and whole brain. Signal-versus- $b$ curves acquired in excised nerves are multi-exponential for diffusion encoding performed both perpendicular and parallel to the nerves [22, $132,133]$. The fast diffusion component has been reported to be almost independent of the diffusion time, while the slow diffusion component has shown evidence of being restricted (Fig. 7). The fast and slow diffusion components were accordingly assigned to the extracellular and intraaxonal spaces, respectively [133]. Estimates of the axon diameter distribution using the AxCaliber model have shown good agreement with corresponding histology-based estimates in porcine optic and sciatic nerves [21]. The estimates were based on several sets of diffusion measurements acquired perpendicular to the nerve and with diffusion times between 10 and $80 \mathrm{~ms}$.

Parameters correlating with the axon diameter can also be obtained using model-free approaches, for example, $q$ space analysis $[66,134,135]$. However, $q$-space analysis underestimates compartment sizes unless $\delta<0.02 d^{2} / D_{\text {intra }}$ [64], which corresponds to $\delta<80 \mu \mathrm{s}$ for $d=2 \mu \mathrm{m}$. Experiments in excised nerves have verified that the compartment size estimated from the slow diffusion component depends on $\delta$ [136], as expected from Eqs. 5 and 6.
Water exchange between the intra-axonal and the extracellular space has been investigated by, for example, Stanisz et al. [22] who modelled nervous tissue as consisting of permeable and uniformly-sized spheres and parallel ellipsoids. The spheres represented glia cells and the ellipsoids represented axons, assuming that the diffusion was restricted also in the direction parallel to the
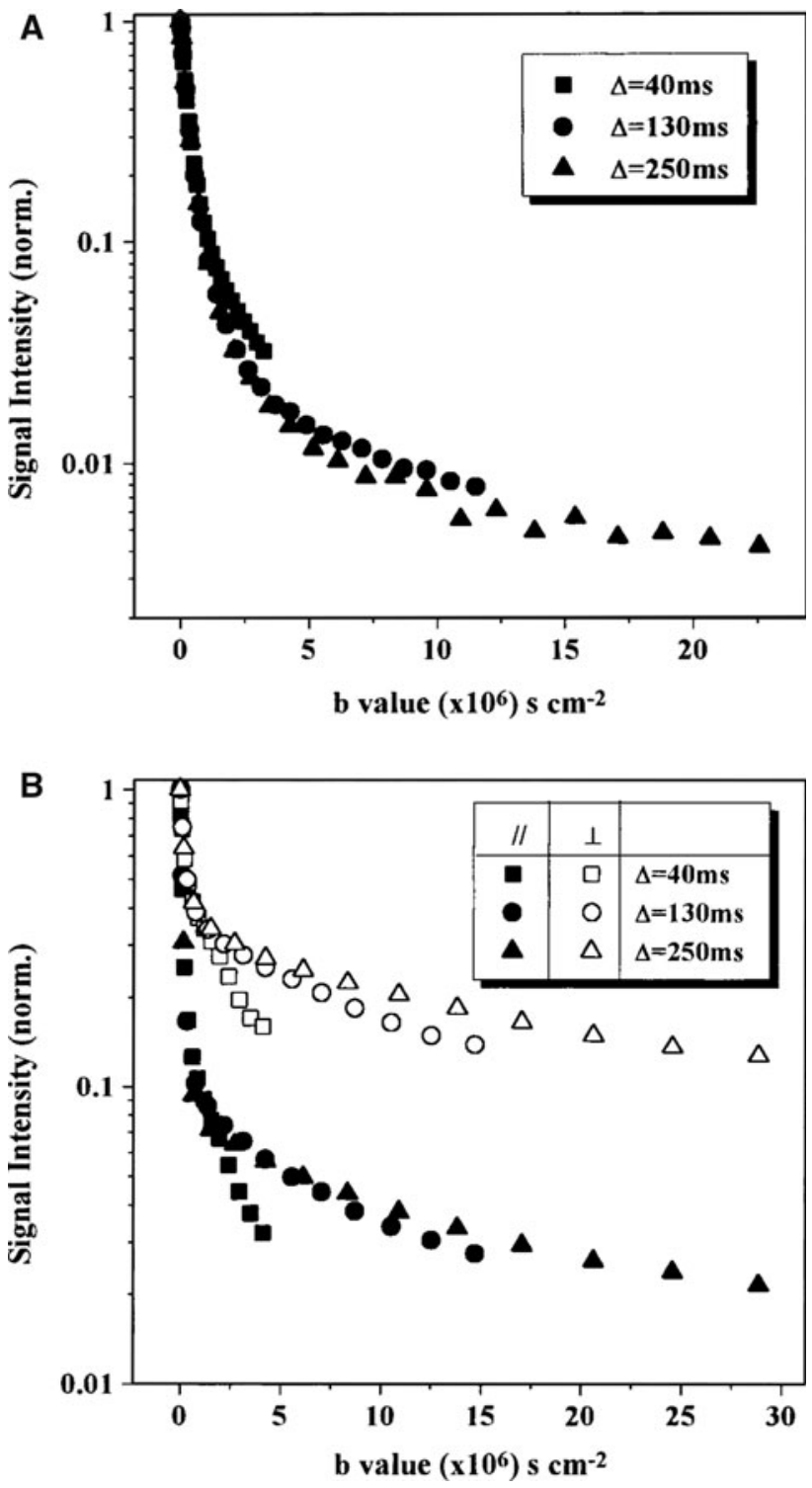

Fig. 7 Normalized attenuation of water signal as a function of the diffusion time, averaged over three brains (a), and three nerves (b). Full and open symbols represent nerve data in which the diffusion gradient direction was parallel (AS) and perpendicular (RS) to the long axis of the nerve, respectively. In the brains (top), the slope of the slow component increase with prolonged diffusion times, while the slope of the slow component is reduced for prolonged diffusion times in nerves (bottom). These two phenomena are the hallmarks of exchange and restricted diffusion, respectively. Note the unit of $b$, where $10^{6} \mathrm{~s} / \mathrm{cm}^{2}=10^{4} \mathrm{~s} / \mathrm{mm}^{2}=10 \mathrm{~ms} / \mu \mathrm{m}^{2}$. Reproduced from Assaf and Cohen [133] with permission from John Wiley and Sons 
axons. Based on measurement in the bovine optic nerve, the authors found that the model required a non-zero membrane permeability (Fig. 8), which was estimated to be $P_{\mathrm{d}}=9 \pm 2$ and $17 \pm 3 \mu \mathrm{m} / \mathrm{s}$ for the axon and glial membrane, respectively. This corresponded to exchange times of approximately $30-60 \mathrm{~ms}$. The axonal and glial water volume fractions were $17 \pm 4$ and $43 \pm 5 \%$, respectively.

Results from other studies also indicate that effects of water exchange are detectable in diffusion-weighted data acquired in excised nerves. Bar-Shir and Cohen performed bi-gaussian analysis of the propagator, similar to biexponential analysis of the signal-versus- $b$ curve, and demonstrated that $f_{\mathrm{s}}$ is reduced as $t_{\mathrm{d}}$ is prolonged above $10 \mathrm{~ms}$ in measurements on the swine optic and sciatic nerves [136]. The observation was attributed partly to water exchange. Biton et al. [137] observed similar trends in normal spinal cord. The authors also investigated myelin-deficient spinal cord, where the root-mean-square displacement of the slow diffusion component increased almost linearly with $\left(t_{\mathrm{d}}\right)^{1 / 2}$, for $t_{\mathrm{d}}$ between 22 and $200 \mathrm{~ms}$. This observation suggests higher exchange rates in the myelin-deficient spinal cord than in the normal one, as could be expected. Assaf et al. [133] observed reduced values of RS at high $b$-values for prolonged diffusion times in the spinal cord of the 7-dayold rat, which is evidence of exchange (Fig. 5). In the mature spinal cord, however, the values of $R S$ increased for prolonged $t_{\mathrm{d}}$, as expected for restricted diffusion. In summary, exchange in excised nerves appears to be fast enough to affect the signal curves acquired so that exchange should be included in models of white matter diffusion. Measuring the exchange rate may be just as important as measuring the axon diameter, since it is altered both in disease and during development.

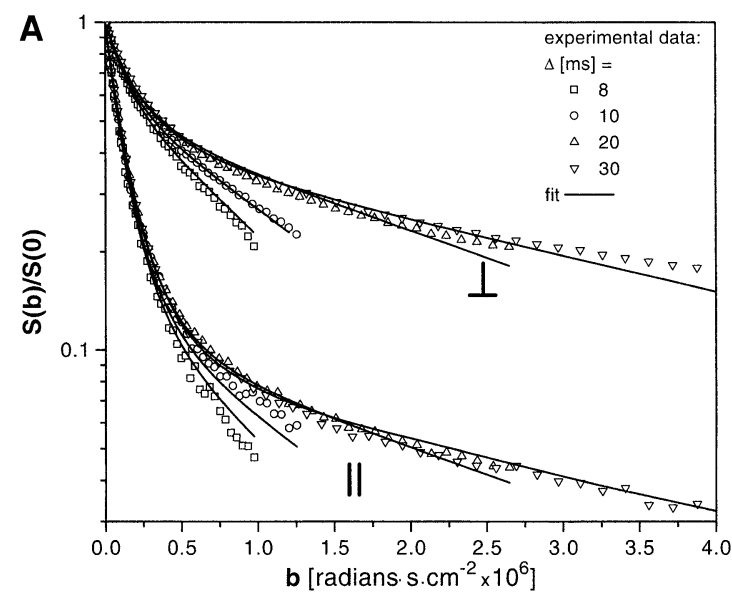

Fig. 8 Signal-versus- $b$ curves obtained with diffusion encoding perpendicular and axial to the bovine optic nerve. a The global fit of a three-pool tissue model (solid lines) to the experimental data (data points). b The results of the three-pool model without

\section{Model validation in vivo}

In contrast to the case in excised tissue, the signal-versus$b$ curves observed in vivo are conspicuously independent of $t_{\mathrm{d}}$, as reported for measurements performed in regions such as the cortex and striatum of the rat as shown in Fig. 9 [53], human white and grey matter, [52] and white matter of the cat [138]. Investigations of RS in the corticospinal tract for diffusion times between 64 and $256 \mathrm{~ms}$ with $b_{\max }=28 \mathrm{~ms} / \mu \mathrm{m}^{2}$ showed no effects of a varied diffusion time (Fig. 10) [23]. At a first glance, these results seem to contradict the assumption that the slow diffusion fraction is restricted, especially since the reported values of $f_{\mathrm{s}}$ are generally much lower than the value of $80 \%$ that would be expected if all intracellular water molecules were restricted in their diffusion. To resolve these issues, it is helpful to analyse white and grey matter separately and to investigate four concepts one by one: differences in relaxivity between excised and living tissue, expected values of the signal fractions, effects of restricted diffusion, and the rate of compartmental exchange.

\section{Relaxivity and diffusion}

Studies of the transversal relaxation in excised nerves have provided evidence of three water components, assigned to myelin water, extracellular water, and intra-axonal water, with $T 2$ relaxation times of $10-20,65-80$, and $250-350 \mathrm{~ms}$, respectively, where the specific values depend on the magnetic field strength [77, 139]. Other studies have suggested that the $T 2$ relaxation time is longer in the extracellular space than in the intra-axonal space $[139,140]$. However, this assignment is not supported by diffusion experiments showing that $f_{\mathrm{r}}$ increases for prolonged TE

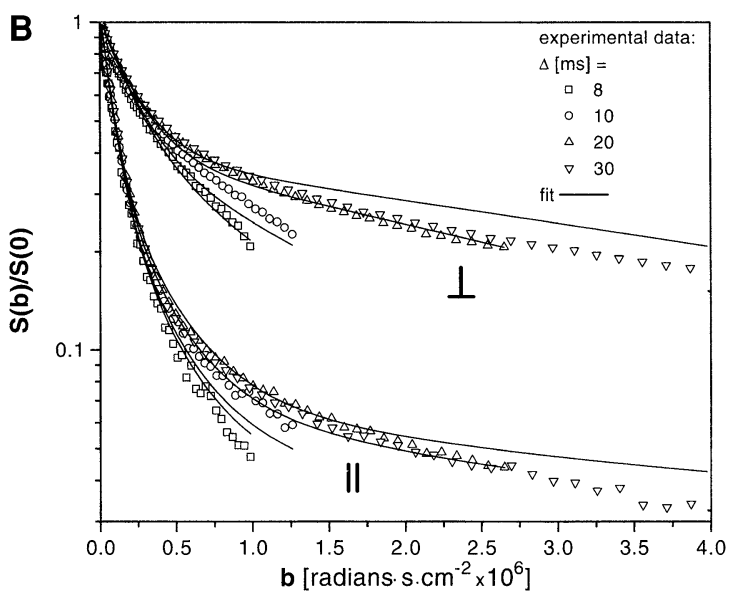

permeability ( $P=0$ for all pools). The misfit for high $b$ values is observable. Note the unit of $b$, where $10^{6} \mathrm{~s} / \mathrm{cm}^{2}=10^{4} \mathrm{~s} / \mathrm{mm}^{2}=$ $10 \mathrm{~ms} / \mu \mathrm{m}^{2}$. Reproduced from Stanisz et al. [22], with permission from John Wiley and Sons 
[77, 133]. Most in vivo studies of transversal relaxation rates have observed two components with short (10-50 ms) and long (70-130 ms) $T 2$ relaxation times, assigned to myelin water and the combined contribution of intra- and extracellular water [141-143]. Support for three components in vivo have been found in the peripheral of the amphibian Xenopus laevis [144], and in some regions in the human brain [141]. Three components could be interpreted as significantly longer $T 2$ relaxation times for extracellular compared to intra-axonal water [142]. However, results from diffusion MRI studies in the CNS suggest at most a

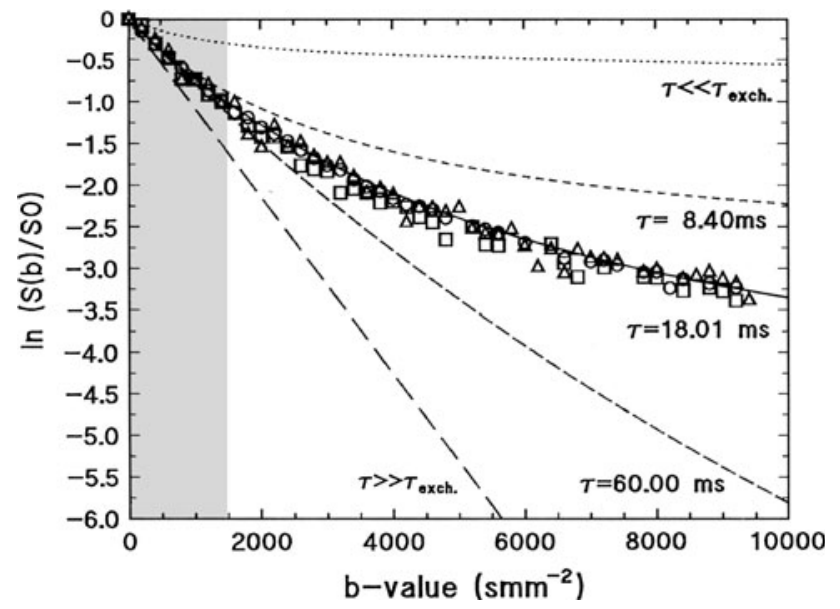

Fig. 9 The plot shows signal attenuation curves obtained in vivo, in the striatum and the cortex of the rat, for three different diffusion times $(8.4,18.01$, and $60 \mathrm{~ms}$ shown by squares, circles, and triangles, respectively). The curves obtained show no diffusion time dependence, in contrast to the dashed curves that would have been expected from the a two-component model (similar to the two-compartment model, but with a fixed diffusion coefficient of the slow component). Note the unit of $b$, where $1 \mathrm{~s} / \mathrm{mm}^{2}=10^{-3} \mathrm{~ms} / \mu \mathrm{m}^{2}$. Reproduced from Niendorf et al. [53], with permission from John Wiley and Sons

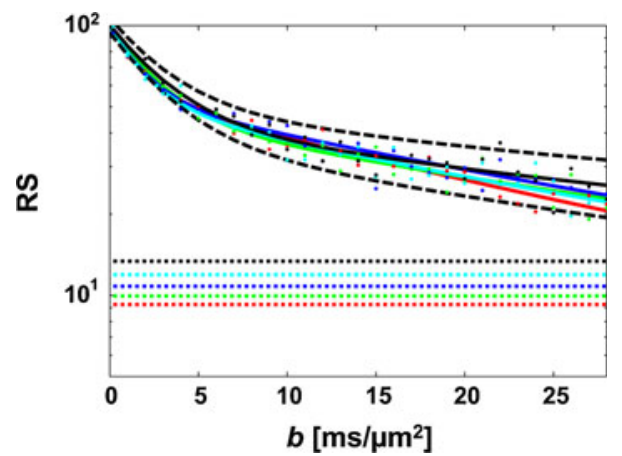

Fig. 10 RS-versus- $b$ curves acquired in the corticospinal tract of a healthy volunteer. Five curves were acquired with $t_{\mathrm{d}}$ from 64 to $256 \mathrm{~ms}$. Solid lines are biexponential fits. No obvious effects of a varied diffusion time is observed in the signal curves. Dashed lines indicate \pm 1 standard deviation of the signal acquired with $t_{\mathrm{d}}=256 \mathrm{~ms}$. Dotted lines show the mean noise level. Reproduced from Nilsson et al. [23], with permission from Elsevier negligible difference in transversal relaxation between the intra-axonal and extracellular space in vivo: the fast- and slow diffusion components have indistinguishable relaxivities [145, 146], DTI metrics are insensitive to TE [147], and biexponential model parameters are insensitive to $\mathrm{TE}$ within practically achievable ranges [52]. These observations suggest that the signal fractions in vivo do reflect the relative volume fractions of the various diffusion components, independent of echo and repetitions times within feasible ranges. Fast exchange between intra-axonal and extracellular water would also render their relaxivities inseparable; however, such a fast exchange is unlikely in healthy white matter.

\section{Signal fractions}

Several authors have performed high $b$-value diffusion experiments in vivo and quantified the resulting signalversus- $b$ curves using a biexponential model. Most of these studies have yielded values for $f_{\mathrm{s}}$ in the range 20-35\% [52, $53,138,148-150]$. This range covers results from varying protocols, acquired in rats as well as in humans and in grey- as well as white matter or a combination of both (Table 2). Values outside this range have been found in studies of white matter where the signal-versus- $b$ curve was acquired in a well-controlled direction compared to the direction of the axons. For instance, Clark and Le Bihan reported $f_{\mathrm{s}} \approx 50 \%$ in the internal capsule, for diffusion encoding performed in the left-right direction [52]. Nilsson et al. [23] similarly reported $f_{\mathrm{s}} \approx 50 \%$ for measurements performed perpendicular to the corticospinal tract.

The hypothesis that the slow diffusion component represents intracellular water has been challenged by the fact that the total intracellular volume fraction $\left(v_{\text {intra }}\right)$ is much higher than the values reported for $f_{\mathrm{s}}[52,149]$. However, intracellular water is distributed in several different environments such as cell bodies of neurons and glial cells as well as in axons and dendrites. Diffusion measured in parallel with axons or dendrites will have a high diffusivity and appear to be unrestricted. Moreover, astrocytic water is probably in fast exchange with the extracellular water, since the ADC is reduced by up to $50 \%$ when the astrocytic AQP4 expression is reduced [49]. Parts of the intracellular water fraction may thus show fast diffusion.

Some intracellular water, such as myelin water, is MRinvisible at the echo times by which most diffusion experiments are performed with clinical MRI scanners. The fractional myelin volume $\left(v_{\text {myelin }}\right)$ may nevertheless have an influence on $f_{\mathrm{r}}$ due to geometrical reasons [55]. Assuming that water compartments other than the intraaxonal, extracellular and myelin compartments are negligible, we have $v_{\text {axon }}+v_{\text {extra }}=1-v_{\text {myelin, }}$, where $v_{\text {extra }}$ here is the fractional volume of the extracellular space and 
other spaces in fast exchange with it. The relation between $v_{\text {axon }}, v_{\text {extra }}$ and $v_{\text {myelin }}$ can be simplified by assuming that the ratio between the axonal outer and inner diameters $(g)$ is independent of the axon diameter (Fig. 1b), and that axons are cylindrical, so that $v_{\text {axon }}=g^{2}\left(v_{\text {axon }}+v_{\text {myelin }}\right)=$ $g^{2}\left(1-v_{\text {extra }}\right)$. Assuming $v_{\text {extra }}=20 \%$ and $g=0.65$ $[40,151]$, the expression for $v_{\text {axon }}$ evaluates to $60 \%$. In the spinal cord, results from segmented histology images suggest that $v_{\text {axon }}$ may be as low as $45 \%$ [135, 152]. Assuming that the water concentrations and relaxivities in the intra-axonal and extracellular spaces are approximately equal, the expected value of $f_{\mathrm{s}}$ may thus be in the range 45-60\%. The presence of axonal orientation dispersion may further reduce the value of $f_{\mathrm{r}}$ [80]. Since $f_{\mathrm{s}} \approx 50 \%$ for diffusion measured perpendicular to white matter [23], it might thus plausible to associate the slow diffusion component to intra-axonal water in white matter, also for the in vivo case. Corresponding analysis of grey matter is more complicated, due to the large dendritic orientation dispersion [153].

\section{Restricted diffusion}

In contrast to what is the case in excised nerves and also expected for restricted diffusion in white matter, the $R S$ versus- $b$ curves obtained in vivo are generally independent of $t_{\mathrm{d}}$ [23, 52, 53, 138]. DTI metrics, obtained in the corpus callosum, are also independent of $t_{\mathrm{d}}$ between 8 and $80 \mathrm{~ms}$ [154]. However, specialised diffusion MRI measurements by Does et al. [155] have revealed a $t_{\mathrm{d}}$ dependence of the ADC for diffusion times below approximately $5 \mathrm{~ms}$. Taken together, these results may imply that $\mathrm{RD}_{\mathrm{r}} \approx 0$ for diffusion times longer than approximately $5 \mathrm{~ms}$. In such cases, the absence of a diffusion-time dependence in RS is to be expected. This is exemplified in Fig. 11, where the compartment model was used to generate $\mathrm{RS}(b)$, assuming $d=6 \mu \mathrm{m}$ and protocols that resemble those employed in NMR spectrometer-based investigations of excised tissue with those used at clinical MRI scanners [23, 67, 133]. While the $t_{\mathrm{d}}$ dependence of the signal is evident for the spectrometer case, it is much weaker for the two cases corresponding to clinical scanners. Specifically, $\mathrm{RD}_{\mathrm{r}} \approx 0$ at both diffusion times in the protocol of Nilsson et al. [23], due to the high value of $\delta$ featured in that protocol. The value of $\delta$ is much shorter in the protocol resembling that employed by Alexander et al., but the low value of $b_{\max }$ results in only a small signal difference between the two diffusion times.

In apparent contradiction with the assumption that $\mathrm{RD}_{\mathrm{r}} \approx 0$, biexponential quantification of signal-versus- $b$ curves shows that $D_{\mathrm{s}}$ is significantly higher than zero in vivo [52, 53, 138, 148-150]. Values of $D_{\text {s }}$ above zero could, however, be expected for measurements performed with the diffusion encoding not being exactly perpendicular to the

Table 2 A summary of the fast and slow ADCs $\left(D_{\mathrm{f}}, D_{\mathrm{s}}\right)$ obtained using the biexponential model in rat and human brains, together with the slow diffusion fraction and details of the protocols employed $\left(t_{\mathrm{d}} / \delta, b_{\max }\right)$

\begin{tabular}{|c|c|c|c|c|c|}
\hline Tissue & Reference & $\mathrm{D}_{\mathrm{f}} / \mathrm{D}_{\mathrm{s}}\left(\mu \mathrm{m}^{2} / \mathrm{ms}\right)$ & $f_{\mathrm{s}}(\%)$ & $t_{\mathrm{d}} / \delta(\mathrm{ms})$ & $b_{\max }\left(\mathrm{ms} / \mu \mathrm{m}^{2}\right)$ \\
\hline Rat brain, $\mathrm{WM}+\mathrm{GM}$ & Niendorf et al. [53] & $0.84 / 0.17$ & 20 & $18 / \mathrm{n} / \mathrm{a}$ & 10 \\
\hline Rat brain, WM & Ronen et al. [138] & $0.69 / 0.08$ & $\sim 30$ & $10 / 8.5$ & 12.5 \\
\hline Rat brain, $\mathrm{WM}+\mathrm{GM}^{\mathrm{a}}$ & Pfeuffer et al. [146] & $0.70 / 0.08$ & $\mathrm{n} / \mathrm{a}$ & $60 / 7$ & 20 \\
\hline Human brain, $\mathrm{WM}^{\mathrm{b}}$ & Clark et al. $[150]^{\mathrm{c}}$ & $0.75 / 0.30$ & 37 & $25 / 27$ & 3.5 \\
\hline Human brain, WM & Clark et al. [52] & $1.12 / 0.16$ & 34 & $25 / \mathrm{n} / \mathrm{a}$ & 4 \\
\hline Human brain, $\mathrm{WM}^{\mathrm{d}}$ & Maier et al. [149] $]^{\mathrm{e}}$ & $1.25 / 0.16$ & 36 & $35 / 35$ & 5 \\
\hline Human brain, WM + GM & Mulkern et al. [148] & $1.40 / 0.25$ & 26 & $56 / 80$ & 6 \\
\hline Human brain, WM & Nilsson et al. [23] & $0.45 / 0.03$ & 51 & $64 / 50$ & 28 \\
\hline Human brain, thalamus & Clark et al. $[150]^{\mathrm{c}}$ & $0.76 / 0.45$ & 37 & $25 / 27$ & 3.5 \\
\hline Human brain, thalamus & Maier et al. [149] ${ }^{\mathrm{e}}$ & $1.18 / 0.23$ & 32 & $35 / 35$ & 5 \\
\hline Mouse cortex & Schwarz et al. [189] & $0.77 / 0.18$ & 21 & $13 / 8$ & 10 \\
\hline Mouse cortex, ischaemic & Schwarz et al. [189] & $0.58 / 0.13$ & 43 & $13 / 8$ & 10 \\
\hline Mouse cortex, cold-injured & Schwarz et al. [189] & $0.89 / 0.10$ & 33 & $13 / 8$ & 10 \\
\hline Adult rat, post mortem & Niendorf et al. [53] & $0.51 / 0.09$ & 31 & $18 / \mathrm{n} / \mathrm{a}$ & 10 \\
\hline
\end{tabular}

Entries are ordered by category and $b_{\max }$

${ }^{a}$ Based on two linear regressions

a Averaged over corpus callosum, the internal capsule, frontal white matter and centrum semiovale

${ }^{c}$ Dual tensor model, based on two linear regressions

d Averaged over the corpus callosum and the internal capsule

e Dual tensor model 
nerve. If $\mathrm{RD}_{\mathrm{r}} \approx 0$ and the encoding direction deviates by an angle $\phi$ from the plane with normal $\mathbf{u}$ (the direction of the nerve), we would expect

$D_{s}=\sin ^{2}(\phi) \mathrm{AD}_{\mathrm{r}}$.

This means that the value of $D_{\mathrm{s}}$ observed by Nilsson et al. [23], using a 3-T head scanner, could have been obtained if $\phi \approx 10^{\circ}$, i.e., if the estimated direction of the nerve deviated more than ten degrees from its true value. Fibre orientation uncertainty can be estimated [156], but are not available for the study. We may however note that such a large deviation appears to be unlikely in a region with high FA [156], which was $0.72 \pm 0.03$ in the region assessed by Nilsson et al. [23]. The high values of $D_{\mathrm{s}}$ observed in vivo probably demands other explanations.

Two other hypotheses could explain the non-zero value of $D_{\mathrm{s}}$ and the apparent absence of a $t_{\mathrm{d}}$-dependence of $\operatorname{RS}(b)$ at high $b$-values. Nilsson et al. [23] suggested that this could be the effect of exchange between the intraaxonal and extracellular space. This hypothesis will be discussed in the next section. Nilsson et al. [80] also described effects of axonal undulation on $\operatorname{RS}(b)$, assuming $\mathrm{RD}_{\mathrm{r}}=0$, and reported that macroscopic undulation results in $t_{\mathrm{d}}$-insensitive and apparently biexponential signal-versus- $b$ curves with non-zero values of $D_{\text {s }}$.

Despite the uncertainties regarding the biophysical mechanism responsible for the slow diffusion component in vivo, estimates of the axon diameter from diffusion MRI data acquired in vivo correlate with corresponding estimates from histology images. Using the AxCaliber model, which assumes impermeable, straight and parallel axons, Barazany et al. [157] estimated the axon diameter distribution from data obtained from the corpus callosum in the rat brain, using a system with $g_{\max }=400 \mathrm{mT} / \mathrm{m}$. The known variations in the axon diameter distribution along the corpus callosum from the anterior (genu) to the posterior (splenium) were largely reproduced, although the axon diameter distributions found by AxCaliber were generally broader than those obtained by histology. The authors suggested that this deviation was caused by tissue shrinkage during histological preparation. Moreover, the reported values of $f_{\mathrm{r}}$ were in the range $15-30 \%$, which is lower than expected. Alexander et al. [67] similarly showed an agreement between the known variations in axon diameter along the corpus callosum and an axon diameter index estimated from diffusion MRI data acquired in two fixed monkey brains and two live volunteers, using an animal experimental system $\left(g_{\max }=140 \mathrm{mT} / \mathrm{m}\right)$ and a clinical MRI scanner $\left(g_{\max }=60 \mathrm{mT} / \mathrm{m}\right)$, respectively. The term "axon diameter index" refers to a summary statistic over the axon diameter distribution that may differ from the volume-weighted average axon diameter, possibly due to non-linear weighting effects when the compartment model assuming a single diameter is used (Fig. 3). The index was, however, overestimated both in the monkey case and the human case, as compared to the value expected from histological investigations.

\section{Exchange}

Several authors have investigated the intracellular exchange time in live brain tissue and reported values between approximately $\tau_{\mathrm{i}}=25$ and $620 \mathrm{~ms}$ [93, 146, 158, 159]. The results were obtained from large volumes containing contributions from a mixture of grey and white matter. Nilsson et al. [23] reported an intra-axonal exchange time of $\tau_{\mathrm{i}}=306 \pm 45 \mathrm{~ms}$ in a well-defined region of the corticospinal tract. Although this value of $\tau_{\mathrm{i}}$ is within the range suggested by previous studies, the analysis did not account for the likely presence of orientation dispersion [23]. The presence of orientation dispersion would probably result in an underestimated value of $\tau_{\mathrm{i}}$ when analysing the data using the two-compartment exchange model, since effects of exchange and of orientation dispersion on RS are similar.

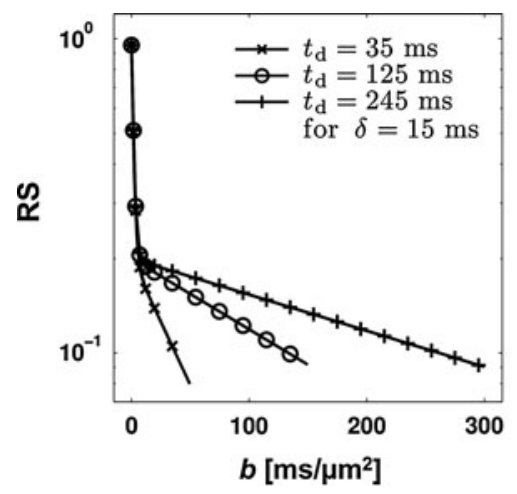

Fig. 11 RS-versus- $b$ curves produced using the compartment model with $f_{\mathrm{r}}=0.2, d=6 \mu \mathrm{m}, \mathrm{RD}_{\mathrm{h}}=0.6 \mu \mathrm{m}^{2} / \mathrm{ms}$. The protocols in the left, middle and right panel intend to resemble the protocols employed
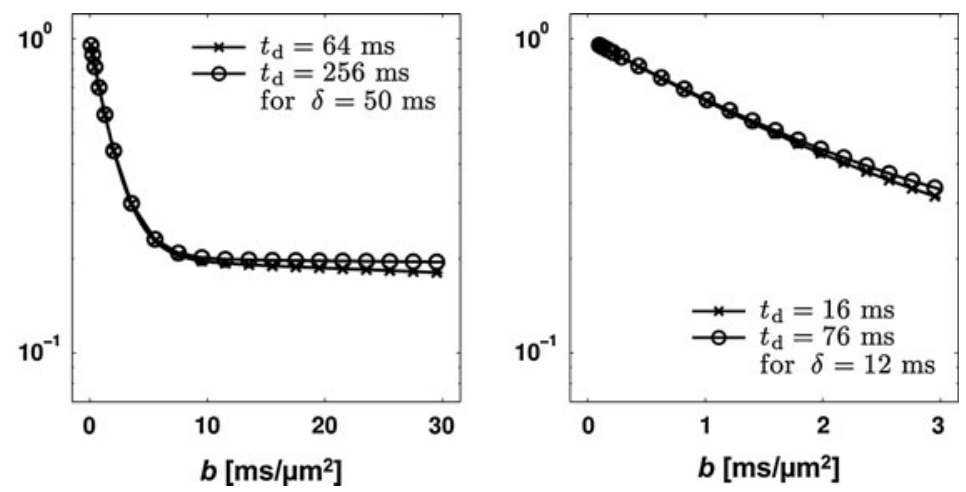

by Assaf et al., by Nilsson et al., and by Alexander et al., respectively. Note the differing scales on the $x$-axis 
The water exchange rate in the human brain has also been investigated using filtered exchange imaging (FEXI), which yields the so-called apparent exchange rate (AXR). In regions of interest placed in frontal and parietal white matter, as well as in the internal capsule, the AXR was $1.6 \pm 0.11,1.0 \pm 0.12$, and $0.8 \pm 0.08 \mathrm{~s}^{-1}$, respectively [103]. These AXR values correspond to exchange times of between 1.25 and $2.5 \mathrm{~s}$, assuming $f_{\mathrm{r}}=50 \%$. These estimated values of $\tau_{\mathrm{i}}$ are considerably longer than those suggested in previous studies.

On the lower part of the exchange-time range observed in the brain, values of $\tau_{\mathrm{i}}$ between 25 and $135 \mathrm{~ms}$ were obtained in grey and white matter regions by Pfeuffer et al. [92, 146] based on constant gradient experiments and reported in two separate studies. These results could suggest the presence of a fast exchanging component with exchange times in the order of 10-100 ms. While the intraaxonal water is presumably in slow exchange with the extracellular space (a slow diffusion component is observed also at long diffusion times), the exchange rate in astrocytes could be high. A reduction of the membrane permeability of these cells, using RNA interference to knockout aquaporin expression, results in ADC reductions of approximately $50 \%$ [49]. Such an effect is only to be expected if the initial exchange rate is high (Fig. 6). Does et al. [160] similarly suggested that one of the components in the $T 2$ spectrum originated from water outside myelinated axons, but within compartments in rapid exchange with the extracellular space.

The rate of water exchange between the intra-axonal and extracellular spaces is probably strongly influenced by the myelin sheath (Fig. 1). For example, studies analysing the relaxivity of different components have suggested that exchange between myelin water and water in the intraaxonal and extracellular space occurs with exchange times of approximately 100-200 ms [143, 161]. Some studies have assumed that the overall permeability of myelin is inversely proportional to the thickness of the myelin sheath $[162,163]$. However, this assumption may only be valid for thin membranes [164]. The intricate structure of the sheath suggests that there could be multiple mechanisms by which the properties of myelin influence the exchange rate. For example, the periaxonal space is connected to the extracellular space, so that water molecules crossing the axolemma can reach the extracellular space without having to pass the myelin membranes (Fig. 1). Another mechanism has been investigated using simulations, in which the myelin was assumed to be impermeable, but where exchange was allowed to take place at the nodes of Ranvier (Fig. 1), as presented in a conference abstract [165]. Describing the nodes by their width $(w)$ and internode distance $(L)$, the ratio of permeable surface to the total volume is given by [103]
$A / V=4 w / \mathrm{d} L$,

which gives an intra-axonal exchange time of $\tau_{\mathrm{i}}=\mathrm{d} L / 4 w P_{\mathrm{d}}$. Although this model is inaccurate for large values of $L$, it may be used to deduce that larger axons with larger distances between the nodes of Ranvier would be expected to show lower exchange rates than thin axons with short distances between the nodes. Future studies could investigate this model by determining the exchange rate in maturing white matter. Simulations suggest that this mechanism would render intra-axonal exchange times in the order of seconds or longer [165], which lends credibility to the idea that intra-axonal water is in slow exchange with the extracellular space.

\section{Application: Ischemic stroke}

Several authors have investigated how DTI parameters are influenced by ischaemic stroke at various stages after onset and hypothesised about the cause of these alterations, as reviewed by Sotak [166]. High $b$-value investigations of diffusion in stroke lesions are less abundant than corresponding DTI studies, but a few studies have quantified the signal-versus- $b$ curve using the biexponential model. Schwarcz et al. [167] showed that $f_{\mathrm{f}}$ decreases in the hyperacute stage of global ischaemia in the mouse brain, as could be expected from the cell-swelling hypothesis that predicts a reduction of the amount of extracellular water in stroke lesions. In addition, both $D_{\mathrm{s}}$ and $D_{\mathrm{f}}$ were reported to decrease as compared to the normal case. Brugières et al. similarly found that $f_{\mathrm{f}}$ decreased in subacute stroke lesions in a patient group, but found that $D_{\mathrm{s}}$ and $D_{\mathrm{f}}$ increased and remained unaffected, respectively [168]. These conflicting results could possibly be explained by the different time from onset in the two studies.

The presence of water exchange between the fast and slow diffusion components complicates the interpretation of results from biexponential analysis of signal-versus$b$ curves. Sub-acute stroke lesions were investigated using high $b$-values and two different diffusion times by Lätt et al. [100]. Effects of exchange were clearly visible in most lesions (Fig. 12). The two-compartment exchange model was fitted to signal curves geometrically averaged across the diffusion-encoding directions, thereby implicitly assuming that the underlying tissue was isotropic. While this assumption is invalid for healthy white matter, the subacute stroke lesions investigated showed lower FA values than healthy tissue. The reduction in anisotropy is also present at high $b$-values, as observed by a reduced kurtosis anisotropy in hyper-acute and acute stroke lesions [169]. By performing an extended analysis of the values presented by Lätt et al. [100], a significant correlation is found 
between $k=1 / \tau_{\mathrm{i}}$ and both FA and MD (Fig. 13). In addition, MD correlated strongly with $f_{\mathrm{h}} D_{\mathrm{h}}(\rho=0.90$, $p=4 \times 10^{-6}$, Spearman), as predicted from Eq. 4. Time from onset, patient age, and $f_{\mathrm{r}}$ did not correlate significantly with any parameter. These correlations suggest that variations in the exchange rate may be responsible for determining the MD and the FA of stroke lesions in the subacute stage. Since MD increases and FA decreases from the early sub-acute stage onwards, a correlation between time from onset and $k$ would have been expected, but it was absent. This absence could possibly be explained by the large heterogeneity in MD and FA observed between patients and within lesions [170, 171]. Follow-up of a cohort of patients with regular measurements could allow this hypothesis to be tested.

Simulations of tissue undergoing ischaemia

Budde and Frank suggested that the total cell surface is preserved when cells swell during ischaemia, which would result in axon and dendrite beading [172]. Monte Carlo simulations of water diffusing in beaded axons showed that this is sufficient to explain a large decrease in AD, MD, and FA. The results were validated by subjecting excised rat sciatic nerve to stretching, which induces beading, but not a bulk shift of water into the axon. The beading mechanism could explain the simultaneous decrease in MD and FA between the hyper-acute and acute stage, but not the simultaneous decrease in FA and increase in MD during the sub-acute stage. However, the latter observation could possibly be explained by exchange as discussed above (Fig. 13).

Other explanations for the reduced MD in stroke have also been suggested based on simulation studies. For instance, Hall and Alexander investigated effects of tissue swelling on the diffusion weighted MRI signal using Monte Carlo simulations and noted that swelling may introduce regions of restricted diffusion in the extracellular space [106]. The authors suggested that this could explain the drastic MD reduction in stroke. In contrast, Jin et al. suggested that cell swelling results in the shrinkage of larger domains in the extracellular space rather than closing of the
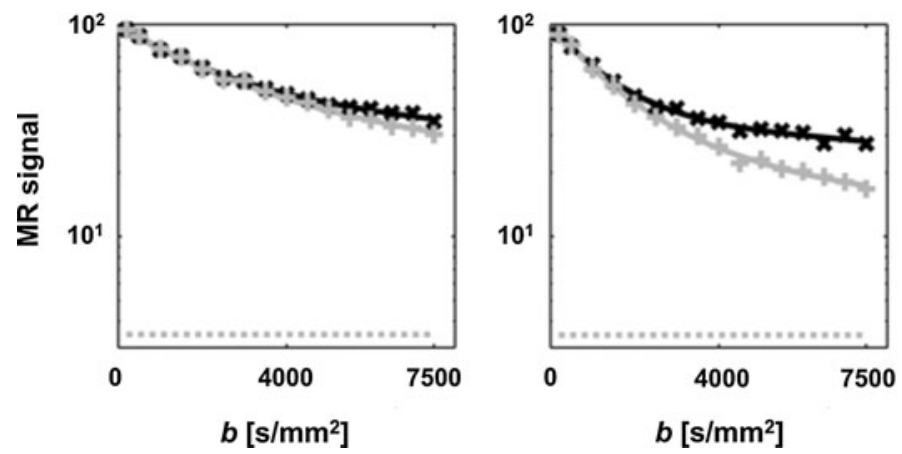

Fig. 12 Signal-versus- $b$ curves obtained with $t_{\mathrm{d}}=60 \mathrm{~ms}$ (black) and $260 \mathrm{~ms}$ (grey), from three regions of interest shown on top of a DWI image on the right, where the white arrow indicates the order of the panels from left to right. Measurements were performed approximately $30 \mathrm{~h}$ after onset. Dashed lines represent the noise floor. Clear
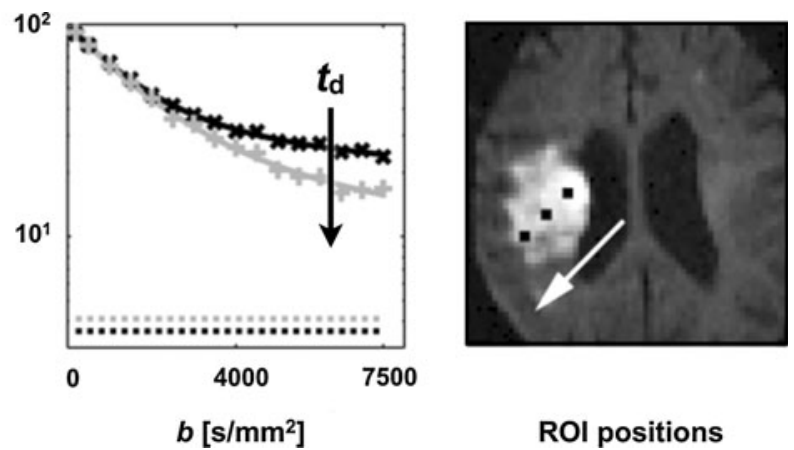

ROI positions

evidence of exchange is seen in the middle and right panel, as reduced signal values for prolonged $t_{\mathrm{d}}$. Note the unit of $b$, where $1 \mathrm{~s} / \mathrm{mm}^{2}=$ $10^{-3} \mathrm{~ms} / \mu \mathrm{m}^{2}$. Reproduced from Lätt et al. [100], with permission from John Wiley and Sons

Fig. 13 Correlation plots for data obtained from Lätt et al. [100], showing the correlation between $k=1 / \tau$, and FA and $\mathrm{MD}$ in the left and right panels, respectively. Correlation was significant for both plots, based on a Spearman correlation test. The solid line is the linear fit
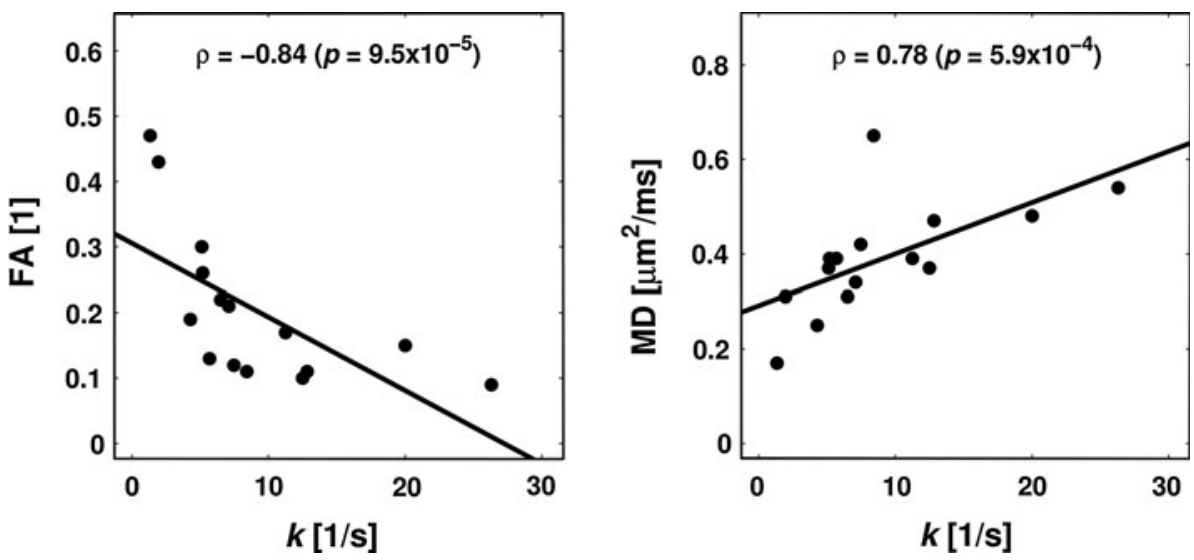
intercellular gap [107]. Harkins et al. [97] reproduced the large reduction in MD observed in stroke by simulating diffusion experiments in a two-compartment system. The large MD reduction was explained by the increase in intracellular volume fraction and by assuming that the $T 2$ relaxation time is much shorter in the intracellular space than in the extracellular space. However, the MD would be highly dependent on TE under such conditions, in contrast to what has been observed experimentally [173].

\section{Altered intracellular diffusivity}

Several authors have tried to perform separate investigations of the intra- and extracellular diffusivities in stroke lesions. For example, Silva et al. [174] measured the ADC in rats where the relaxivity was selectively enhanced in the extracellular space, and tuned the echo time so that only intracellular signal contributed to the measured ADC value. No major differences in the ADC values were observed between normal measurements and relaxation-enhanced measurements, indicating that there is no difference in ADC between the intracellular and extracellular space, or that the extracellular signal fraction is negligible. Following middle cerebral artery occlusion, the ADC was reduced by approximately $40 \%$, for both the normal and the relaxation-enhanced measurement. The reduction in $D_{\text {intra }}$ following ischemia is also supported by reports of a reduced value of $D_{\text {intra }}$ immediately after death, as observed in diffusion experiments with sub-millisecond diffusion times achieved by the use of oscillating gradients [155]. Doung et al. [175] determined the ADC of intracellular- and extracellular-specific molecular markers and did not detect any difference in ADC in the two spaces. Similarly, Neil et al. reported that the ADC of ${ }^{133} \mathrm{Cs}$ that accumulated intracellularly was reduced in global brain ischaemia.

While these studies do suggest that $D_{\text {intra }}$ is reduced following an ischaemic stroke, it is not clear what to expect regarding $D_{\mathrm{r}}$. Reduction of $D_{\mathrm{i}}$ leads to lower values of $\alpha$ and $\beta$ (Eq. 6), which could actually result in increased values of $D_{\mathrm{r}}$ (Fig. 2). Separate measurements acquired with varying diffusion times would be required to better understand the implications of reduced values of $D_{\text {intra }}$ on metrics observed by conventional diffusion MRI.

\section{Other applications}

Conventional DTI has numerous clinical applications [176], and biophysical modelling of diffusion in white matter can help understand the mechanisms underlying alterations in DTI parameters. For example, Sen and Basser concluded that MD and FA are primarily influenced by changes in the outer diameter of axons, the extracellular volume fraction and the inter-axonal spacing [177]. Harkins et al. [97] used simulations to show that the ADC is nearly insensitive to variations in the membrane permeability. Nilsson et al. [80] suggested that stretching of nerves composed of undulating axons could increase the FA, based on results from simulations. Despite the progress made by such modelling studies, two shortcomings intrinsic in DTI remain: that the resulting parameters only indirectly related to the tissue microstructure [178], and that results can be confounded by the presence of crossing fibres and partial volume effects [179-181]. Due to such shortcomings, DTI results must be carefully scrutinized to avoid the misinterpretation that follows if FA interpreted is a measure of "white matter integrity" [182]. This claim is exemplified by the counterintuitive finding of elevated FA in a region of the brain of patients with mild cognitive impairment [183]. This result was interpreted as the relative sparing of motor-related pathways compared to cognitive-related ones in areas of crossing fibres, resulting in an increased homogeneity of fibre orientations.

To solve the problems intrinsic in DTI, biophysical models can be used to extract parameters more specific to the tissue microstructure, from data acquired with extended protocols. Such extended protocols may feature $b$-values higher than those used in DTI, which allows for crossing fibers to be resolved $[179,184]$. Acquiring data with higher $b$-values may also increase the sensitivity to tissue microstructure alterations, which was explored in early studies of diseases such as multiple sclerosis [185], vascular dementia [186], and to follow up of treatment in intracranial tumours [187]. However, model-based assessment of microstructural properties such as the axon diameter or the intracellular exchange time require data to be acquired not only with higher $b$-values than in DTI, but also with variable diffusion times. Acquisition of such data comes with a price: longer scan times. This problem can be partially solved by improved pulse sequence design, as in the case of filter exchange imaging [87, 103], by using algorithmic protocol design [67], or by relevant simplifications of complex models as in the case of NODDI [82]. As many of the models and strategies described in this review have only recently been developed, their clinical applications are yet scarce, but that may change in the near future due to recent improvements in hardware and data acquisition strategies [188].

\section{Conclusions}

Biophysical models of diffusion in white matter have been constructed to include effects of restricted diffusion in approximately cylindrical axons, a distribution of axon 
diameters, orientation dispersion and exchange between the intra-axonal and extra-axonal space, allowing these properties to be inferred from diffusion MRI experiments. In practise, however, properties such as parameters of the axon diameter distribution may be possible to estimate accurately in vivo only if limitations in the scanner hardware are overcome, most notably, limited values of $g_{\max }$. In general, the predictions made based on biophysical models agree with experiments performed in vivo. For example, the value of the signal fraction of slowly diffusing water agrees with the expected, assuming that myelin water is invisible at the long echo times at which diffusion MRI is performed. The specific tissue properties that determine the characteristics of the slowly diffusing water are, however, not yet fully characterized, although the water exchange rate and degree of orientation dispersion probably both contribute. Accordingly, those tissue properties also affect parameters determined using models such as DTI and DKI. For example, MD and FA are probably influenced by the degree of axonal undulation in extracranial nerves, while they correlate with the exchange rate in subacute stroke lesions. Recent studies have also shown that three-dimensional properties of white matter are required to take into account in order to further understand how the tissue properties affect the outcome of diffusion MRI experiments.

Acknowledgments The study was supported by the Swedish Cancer Society (Grant No. CAN 2009/1076 and 2012/597) and the Swedish Research Council (Grant No. 521-210-3034).

Open Access This article is distributed under the terms of the Creative Commons Attribution License which permits any use, distribution, and reproduction in any medium, provided the original author(s) and the source are credited.

\section{References}

1. Minati L, Weglarz W (2007) Physical foundations, models, and methods of diffusion magnetic resonance imaging of the brain: a review. Concepts Magn Reson Part A Bridge Educt Res 30(5): 278-307

2. Price W (1997) Pulsed-field gradient nuclear magnetic resonance as a tool for studying translational diffusion: part 1. Basic theory. Concepts Magn Reson Part A Bridge Educt Res 9: 299-336

3. Stejskal EO, Tanner JE (1965) Spin diffusion measurements: spin echoes in the presence of a time-dependent field gradient. J Chem Phys 42(1):288-292

4. Stejskal EO (1965) Use of spin echoes in a pulsed magnetic-field gradient to study anisotropic, restricted diffusion and flow. J Chem Phys 43:3597

5. Cory DG, Garroway AN, Miller JB (1990) Applications of spin transport as a probe of local geometry. Polym Prepr 31:149

6. Mitra P (1995) Multiple wave-vector extensions of the NMR pulsed-field-gradient spin-echo diffusion measurement. Phys Rev B 51(21):15074-15078
7. Callaghan P, Komlosh M (2002) Locally anisotropic motion in a macroscopically isotropic system: displacement correlations measured using double pulsed gradient spin-echo NMR. Magn Reson Chem 40(13):S15-S19

8. Komlosh ME, Horkay F, Freidlin RZ, Nevo U, Assaf Y, Basser PJ (2007) Detection of microscopic anisotropy in gray matter and in a novel tissue phantom using double pulsed gradient spin echo MR. J Magn Reson 189(1):38-45

9. Özarslan E, Basser PJ (2008) Microscopic anisotropy revealed by NMR double pulsed field gradient experiments with arbitrary timing parameters. J Chem Phys 128(15):154511

10. Shemesh N, Ozarslan E, Komlosh ME, Basser PJ, Cohen Y (2010) From single-pulsed field gradient to double-pulsed field gradient MR: gleaning new microstructural information and developing new forms of contrast in MRI. NMR Biomed 23(7): 757-780

11. Koch MA, Finsterbusch J (2008) Compartment size estimation with double wave vector diffusion-weighted imaging. Magn Reson Med 60(1):90-101

12. Callaghan PT, Furó I (2004) Diffusion-diffusion correlation and exchange as a signature for local order and dynamics. J Chem Phys 120(8):4032-4038

13. Åslund I, Nowacka A, Nilsson M, Topgaard D (2009) Filterexchange PGSE NMR determination of cell membrane permeability. J Magn Reson 200(2):291-295

14. Callaghan PT, Stepisnik J (1995) Frequency-domain analysis of spin motion using modulated-gradient NMR. J Magn Reson A 117(1):118-122

15. Gross B, Kosfeld R (1969) Anwendung der spin-echo-methode der messung der selbstdiffusion. Messtechnik 77:171-177

16. Stepisnik J (1981) Analysis of NMR self-diffusion measurements by a density matrix calculation. Phys B 104(3):350-364

17. Schachter M, Does MD, Anderson AW, Gore JC (2000) Measurements of restricted diffusion using an oscillating gradient spin-echo sequence. J Magn Reson 147(2):232-237

18. Drobnjak I, Siow B, Alexander DC (2010) Optimizing gradient waveforms for microstructure sensitivity in diffusion-weighted MR. J Magn Reson 206(1):41-51

19. Alexander DC (2008) A general framework for experiment design in diffusion MRI and its application in measuring direct tissue-microstructure features. Magn Reson Med 60(2):439-448

20. Assaf Y, Freidlin RZ, Rohde GK, Basser PJ (2004) New modeling and experimental framework to characterize hindered and restricted water diffusion in brain white matter. Magn Reson Med 52(5):965-978

21. Assaf Y, Blumenfeld-Katzir T, Yovel Y, Basser PJ (2008) AxCaliber: a method for measuring axon diameter distribution from diffusion MRI. Magn Reson Med 59(6):1347-1354

22. Stanisz GJ, Szafer A, Wright GA, Henkelman M (1997) An analytical model of restricted diffusion in bovine optic nerve. Magn Reson Med 37:103-111

23. Nilsson M, Lätt J, Nordh E, Wirestam R, Ståhlberg F, Brockstedt $S$ (2009) On the effects of a varied diffusion time in vivo: is the diffusion in white matter restricted? Magn Reson Imaging 27(2):176-187

24. Basser PJ, Mattiello J, LeBihan D (1994) Estimation of the effective self-diffusion tensor from the NMR spin echo. J Magn Reson B 103:247-254

25. Liu C, Bammer R, Acar B, Moseley ME (2004) Characterizing non-gaussian diffusion by using generalized diffusion tensors. Magn Reson Med 51(5):924-937

26. Jensen JH, Helpern JA, Ramani A, Lu H, Kaczynski K (2005) Diffusional kurtosis imaging: the quantification of non-gaussian water diffusion by means of magnetic resonance imaging. Magn Reson Med 53(6):1432-1440 
27. Jensen JH, Helpern JA (2010) MRI quantification of nongaussian water diffusion by kurtosis analysis. NMR Biomed 23(7):698-710

28. Bennett KM, Schmainda KM, Bennett RT, Rowe DB, Lu H, Hyde JS (2003) Characterization of continuously distributed cortical water diffusion rates with a stretched-exponential model. Magn Reson Med 50(4):727-734

29. Yablonskiy DA, Bretthorst GL, Ackerman JJH (2003) Statistical model for diffusion attenuated MR signal. Magn Reson Med 50(4):664-669

30. Fieremans E, Jensen JH, Helpern JA (2011) White matter characterization with diffusional kurtosis imaging. Neuroimage 58(1):177-188

31. Lätt J, Nilsson M, Rydhög A, Wirestam R, Ståhlberg F, Brockstedt S (2007) Effects of restricted diffusion in a biological phantom: a q-space diffusion MRI study of asparagus stems at a 3T clinical scanner. Magn Reson Mater Phy 20(4):213-222

32. Lätt J, Nilsson M, Malmborg C, Rosquist H, Wirestam R, Ståhlberg F, Topgaard D, Brockstedt S (2007) Accuracy of q-space related parameters in MRI: simulations and phantom measurements. IEEE Trans Med Imaging 26(11):1437-1447

33. Edgar JM, Griffiths IR (2009) White matter structure: a microscopist's view. In: Diffusion MRI from quantitative measurement to neuroanatomy, Academic Press, pp 73-103

34. Allen NJ, Barres BA (2009) Neuroscience: glia-more than just brain glue. Nature 457(7230):675-677

35. Beaulieu C (2002) The basis of anisotropic water diffusion in the nervous system-a technical review. NMR Biomed 15(7-8): $435-455$

36. Aboitiz F, Scheibel AB, Fisher RS, Zaidel E (1992) Fiber composition of the human corpus callosum. Brain Res 598(1-2): 143-153

37. Tang Y, Nyengaard JR, Pakkenberg B, Gundersen HJ (1997) Age-induced white matter changes in the human brain: a stereological investigation. Neurobiol Aging 18(6):609-615

38. Michailov GV, Sereda MW, Brinkmann BG, Fischer TM, Haug B, Birchmeier C, Role L, Lai C, Schwab MH, Nave K-A (2004) Axonal neuregulin-1 regulates myelin sheath thickness. Science 304(5671):700-703

39. Sturrock RR (1980) Myelination of the mouse corpus callosum. Neuropathol Appl Neurobiol 6(6):415-420

40. Paus T (2010) Growth of white matter in the adolescent brain: myelin or axon? Brain Cognit 72(1):26-35

41. Waxman SG, Bennett MV (1972) Relative conduction velocities of small myelinated and non-myelinated fibres in the central nervous system. Nat New Biol 238(85):217-219

42. Rushton WAH (1951) A theory of the effects of fibre size in medullated nerve. J Physiol (Lond) 115(1):101-122

43. Hildebrand C, Remahl S, Persson H, Bjartmar C (1993) Myelinated nerve fibres in the CNS. Prog Neurobiol 40(3):319-384

44. Hursh JB (1939) Conduction velocity and diameter of nerve fibers. Am J Physiol 127:131-139

45. Perge JA, Koch K, Miller R, Sterling P, Balasubramanian V (2009) How the optic nerve allocates space, energy capacity, and information. J Neurosci 29(24):7917-7928

46. Sunderland S (1965) The connective tissues of peripheral nerves. Brain 88(4):841-854

47. Breig A (1960) Biomechanics of the central nervous system: some basic normal and pathologic phenomena. Almqvist \& Wiksell, Stockholm

48. Jeffery G (1996) PNS features of rodent optic nerve axons. J Comp Neurol 366(2):370-378

49. Badaut J, Ashwal S, Adami A, Tone B, Recker R, Spagnoli D, Ternon B, Obenaus A (2011) Brain water mobility decreases after astrocytic aquaporin-4 inhibition using RNA interference. J Cereb Blood Flow Metab 31(3):819-831
50. Tait MJ, Saadoun S, Bell BA, Papadopoulos MC (2008) Water movements in the brain: role of aquaporins. Trends Neurosci 31(1):37-43

51. Castle NA (2005) Aquaporins as targets for drug discovery. Drug Discov Today 10(7):485-493

52. Clark CA, Le Bihan D (2000) Water diffusion compartmentation and anisotropy at high $\mathrm{b}$ values in the human brain. Magn Reson Med 44(6):852-859

53. Niendorf T, Dijkhuizen RM, Norris DG, van Lookeren Campagne M, Nicolay K (1996) Biexponential diffusion attenuation in various states of brain tissue: implications for diffusionweighted imaging. Magn Reson Med 36(6):847-857

54. Moseley ME, Cohen Y, Kucharczyk J, Mintorovitch J, Asgari HS, Wendland MF, Tsuruda J, Norman D (1990) Diffusionweighted MR imaging of anisotropic water diffusion in cat central nervous system. Radiology 176(2):439-445

55. Peled S (2007) New perspectives on the sources of white matter DTI signal. IEEE Trans Med Imaging 26(11):1448-1455

56. Beaulieu C, Allen PS (1994) Water diffusion in the giant axon of the squid: implications for diffusion-weighted MRI of the nervous system. Magn Reson Med 32(5):579-583

57. Beaulieu C, Allen PS (1996) An in vitro evaluation of the effects of local magnetic-susceptibility-induced gradients on anisotropic water diffusion in nerve. Magn Reson Med 36(1):39-44

58. Frøhlich AF, Jespersen SN, Ostergaard L, Kiselev VG (2008) The effect of impermeable boundaries of arbitrary geometry on the apparent diffusion coefficient. J Magn Reson 194(1): $128-135$

59. Padhani AR, Liu G, Koh DM et al (2009) Diffusion-weighted magnetic resonance imaging as a cancer biomarker: consensus and recommendations. Neoplasia 11(2):102-125

60. Murday J, Cotts R (1968) Self-diffusion coefficient of liquid lithium. J Chem Phys 48:4938

61. Stepisnik J (1993) Time-dependent self-diffusion by NMR spinecho. Phys B 183(4):343-350

62. Neuman C (1974) Spin echo of spins diffusing in a bounded medium. J Chem Phys 60:4508

63. van Gelderen P, DesPres D, van Zijl PCM, Moonen CT (1994) Evaluation of restricted diffusion in cylinders. Phosphocreatine in rabbit leg muscle. J Magn Reson B 103(3):255-260

64. Wang L, Caprihan A, Fukushima E (1995) The narrow-pulse criterion for pulsed-gradient spin-echo diffusion measurements. J Magn Reson A 117(2):209-219

65. Zhang H, Barazany D, Assaf Y, Lundell HM, Alexander DC, Dyrby TB (2011) A comparative study of axon diameter imaging techniques using diffusion MRI. Proc Intl Soc Mag Reson Med 19:83

66. Cohen Y, Assaf Y (2002) High b-value q-space analyzed diffusion-weighted MRS and MRI in neuronal tissues - a technical review. NMR Biomed 15(7-8):516-542

67. Alexander DC, Hubbard PL, Hall MG, Moore EA, Ptito M, Parker GJM, Dyrby TB (2010) Orientationally invariant indices of axon diameter and density from diffusion MRI. Neuroimage 52(4):1374-1389

68. Nilsson M, Alerstam E, Wirestam R, Ståhlberg F, Brockstedt S, Lätt J (2010) Evaluating the accuracy and precision of a twocompartment Kärger model using Monte Carlo simulations. J Magn Reson 206(1):59-67

69. Nilsson M, Alexander DC (2012) Investigating tissue microstructure using diffusion MRI: how does the resolution limit of the axon diameter relate to the maximal gradient strength? Proc Intl Soc Mag Reson Med 20:3567

70. Balinov B, Jonsson B, Linse P, Söderman O (1993) The NMR self-diffusion method applied to restricted diffusion. simulation of echo attenuation from molecules in spheres and between planes. J Magn Reson A 104(1):17-25 
71. Callaghan P, Coy A, MacGowan D, Packer K, Zelaya F (1991) Diffraction-like effects in NMR diffusion studies of fluids in porous solids. Nature 351:467-469

72. Avram L, Assaf Y, Cohen Y (2004) The effect of rotational angle and experimental parameters on the diffraction patterns and micro-structural information obtained from q-space diffusion NMR: implication for diffusion in white matter fibers. J Magn Reson 169(1):30-38

73. Avram L, Ozarslan E, Assaf Y, Bar-Shir A, Cohen Y, Basser PJ (2008) Three-dimensional water diffusion in impermeable cylindrical tubes: theory versus experiments. NMR Biomed 21(8):888-898

74. Lennon AJ, Kuchel PW (1994) Enhancement of the diffractionlike effect in NMR diffusion experiments. J Magn Reson A 111(2):208-211

75. Topgaard D, Söderman O (2003) Experimental determination of pore shape and size using q-space NMR microscopy in the long diffusion-time limit. Magn Reson Imaging 21(1):69-76

76. Yablonskiy DA, Sukstanskii AL (2010) Theoretical models of the diffusion weighted MR signal. NMR Biomed 23(7):661-681

77. Peled S, Cory DG, Raymond SA, Kirschner DA, Jolesz FA (1999) Water diffusion, $\mathrm{T}(2)$, and compartmentation in frog sciatic nerve. Magn Reson Med 42(5):911-918

78. Jespersen SN, Pedersen M, Stødkilde-Jørgensen H (2005) The influence of a cellular size distribution on NMR diffusion measurements. Eur Biophys J 34(7):890-898

79. Leergaard TB, White NS, De Crespigny A, Bolstad I, D'arceuil H, Bjaalie JG, Dale AM (2010) Quantitative histological validation of diffusion MRI fiber orientation distributions in the rat brain. PLoS ONE 5(1):e8595

80. Nilsson M, Lätt J, Ståhlberg F, van Westen D, Hagslätt H (2012) The importance of axonal undulation in diffusion MR measurements: a Monte Carlo simulation study. NMR Biomed 25(5):795-805

81. Zhang H, Hubbard PL, Parker GJM, Alexander DC (2011) Axon diameter mapping in the presence of orientation dispersion with diffusion MRI. Neuroimage. doi:10.1016/j.neuroimage.2011. 01.084

82. Zhang H, Schneider T, Wheeler-Kingshott CA, Alexander DC (2012) NODDI: practical in vivo neurite orientation dispersion and density imaging of the human brain. Neuroimage 61(4):1000-1016

83. Karami G, Grundman N, Abolfathi N, Naik A, Ziejewski M (2009) A micromechanical hyperelastic modeling of brain white matter under large deformation. J Mech Behav Biomed Mater 2(3):243-254

84. Kärger J (1971) Der Einfluß der Zweibereichdiffusion auf die Spinechodämpfung unter Berücksichtigung der relaxation bei Messungen mit der Methode der gepulsten Feldgradienten. Ann Phys 482(1):107-109

85. Kärger J (1969) Zur Bestimmung der Diffusion in einem Zweibereichsystem mit Hilfe von gepulsten Feldgradienten. Ann Phys 479(1-2):1-4

86. Fieremans E, Novikov DS, Jensen JH, Helpern JA (2010) Monte Carlo study of a two-compartment exchange model of diffusion. NMR Biomed 23(7):711-724

87. Lasič S, Nilsson M, Lätt J, Ståhlberg F, Topgaard D (2011) Apparent exchange rate mapping with diffusion MRI. Magn Reson Med 66(2):356-365

88. Jansen M, Blume A (1995) A comparative study of diffusive and osmotic water permeation across bilayers composed of phospholipids with different head groups and fatty acyl chains. Biophys J 68(3):997-1008

89. Ye RG, Verkman AS (1989) Simultaneous optical measurement of osmotic and diffusional water permeability in cells and liposomes. Biochemistry 28(2):824-829
90. Verkman AS (2000) Water permeability measurement in living cells and complex tissues. J Membr Biol 173(2):73-87

91. Benga G (1988) Water transport in red blood cell membranes. Prog Biophys Mol Biol 51(3):193-245

92. Pfeuffer J, Flögel U, Dreher W, Leibfritz D (1998) Restricted diffusion and exchange of intracellular water: theoretical modelling and diffusion time dependence of $1 \mathrm{H}$ NMR measurements on perfused glial cells. NMR Biomed 11(1):19-31

93. Meier C, Dreher W, Leibfritz D (2003) Diffusion in compartmental systems. I. A comparison of an analytical model with simulations. Magn Reson Med 50(3):500-509

94. Lee J-H, Springer CS (2003) Effects of equilibrium exchange on diffusion-weighted NMR signals: the diffusigraphic "shutterspeed". Magn Reson Med 49(3):450-458

95. Regan DG, Kuchel PW (2000) Mean residence time of molecules diffusing in a cell bounded by a semi-permeable membrane: Monte Carlo simulations and an expression relating membrane transition probability to permeability. Eur Biophys J 29:221-227

96. Sehy JV, Banks AA, Ackerman JJH, Neil JJ (2008) Importance of intracellular water apparent diffusion to the measurement of membrane permeability. Biophys J 83(5):2856-2863

97. Harkins KD, Galons J-P, Secomb TW, Trouard TP (2009) Assessment of the effects of cellular tissue properties on ADC measurements by numerical simulation of water diffusion. Magn Reson Med 62(6):1414-1422

98. Behrens TEJ, Berg HJ, Jbabdi S, Rushworth MFS, Woolrich MW (2007) Probabilistic diffusion tractography with multiple fibre orientations: what can we gain? Neuroimage 34(1):144-155

99. Jeurissen B, Leemans A, Tournier J-D, Jones DK, Sijbers J (2012) Investigating the prevalence of complex fiber configurations in white matter tissue with diffusion magnetic resonance imaging. Hum Brain Mapp. doi:10.1002/hbm.22099

100. Lätt J, Nilsson M, Van Westen D, Wirestam R, Ståhlberg F, Brockstedt $S$ (2009) Diffusion-weighted MRI measurements on stroke patients reveal water-exchange mechanisms in sub-acute ischaemic lesions. NMR Biomed 22(6):619-628

101. Kiselev VG, Il'yasov KA (2007) Is the "biexponential diffusion" biexponential? Magn Reson Med 57(3):464-469

102. Panagiotaki E, Schneider T, Siow B, Hall MG, Lythgoe MF, Alexander DC (2012) Compartment models of the diffusion MR signal in brain white matter: a taxonomy and comparison. Neuroimage 59(3):2241-2254

103. Nilsson M, Lätt J, van Westen D, Brockstedt S, Lasič S, Ståhlberg F, Topgaard D (2012) Noninvasive mapping of water diffusional exchange in the human brain using filter-exchange imaging. Magn Reson Med. doi:10.1002/mrm.24395

104. Nicholson C, Phillips JM (1981) Ion diffusion modified by tortuosity and volume fraction in the extracellular microenvironment of the rat cerebellum. J Physiol 321:225-257

105. Lipinski H (1990) Monte Carlo simulation of extracellular diffusion in brain tissues. Phys Med Biol 35:441

106. Hall M, Alexander DC (2009) Convergence and parameter choice for Monte-Carlo simulations of diffusion MRI. IEEE Trans Med Imaging 28(9):1354-1364

107. Jin S, Zador Z, Verkman AS (2008) Random-walk model of diffusion in three dimensions in brain extracellular space: comparison with microfiberoptic photobleaching measurements. Biophys J 95(4):1785-1794

108. Jones DK, Cercignani M (2010) Twenty-five pitfalls in the analysis of diffusion MRI data. NMR Biomed 23(7):803-820

109. Le Bihan D, Poupon C, Amadon A, Lethimonnier F (2006) Artifacts and pitfalls in diffusion MRI. J Magn Reson Imaging 24(3):478-488

110. Bernstein MA, Thomasson DM, Perman WH (1989) Improved detectability in low signal-to-noise ratio magnetic resonance 
images by means of a phase-corrected real reconstruction. Med Phys 16(5):813-817

111. Gudbjartsson H, Patz S (1995) The Rician distribution of noisy MRI data. Magn Reson Med 34(6):910-914

112. Veraart J, Van Hecke W, Sijbers J (2011) Constrained maximum likelihood estimation of the diffusion kurtosis tensor using a Rician noise model. Magn Reson Med 66(3):678-686

113. Constantinides CD, Atalar E, McVeigh ER (1997) Signal-tonoise measurements in magnitude images from NMR phased arrays. Magn Reson Med 38(5):852-857

114. Dietrich O, Raya JG, Reeder SB, Ingrisch M, Reiser MF, Schoenberg SO (2008) Influence of multichannel combination, parallel imaging and other reconstruction techniques on MRI noise characteristics. Magn Reson Imaging 26(6):754-762

115. Veraart J, Rajan J, Peeters RR, Leemans A, Sunaert S, Sijbers J (2012) Comprehensive framework for accurate diffusion MRI parameter estimation. Magn Reson Med. doi:10.1002/mrm. 24529

116. Pruessmann KP, Weiger M, Scheidegger MB, Boesiger P (1999) SENSE: sensitivity encoding for fast MRI. Magn Reson Med 42(5):952-962

117. Thelwall PE, Shepherd TM, Stanisz GJ, Blackband SJ (2006) Effects of temperature and aldehyde fixation on tissue water diffusion properties, studied in an erythrocyte ghost tissue model. Magn Reson Med 56(2):282-289

118. D'arceuil H, De Crespigny A (2007) The effects of brain tissue decomposition on diffusion tensor imaging and tractography. Neuroimage 36(1):64-68

119. Sun S-W, Neil JJ, Liang H-F, He YY, Schmidt RE, Hsu CY, Song S-K (2005) Formalin fixation alters water diffusion coefficient magnitude but not anisotropy in infarcted brain. Magn Reson Med 53(6):1447-1451

120. Schmierer K, Wheeler-Kingshott CAM, Tozer DJ, Boulby PA, Parkes HG, Yousry TA, Scaravilli F, Barker GJ, Tofts PS, Miller DH (2008) Quantitative magnetic resonance of postmortem multiple sclerosis brain before and after fixation. Magn Reson Med 59(2):268-277

121. Dyrby TB, Baaré WFC, Alexander DC, Jelsing J, Garde E, Søgaard LV (2011) An ex vivo imaging pipeline for producing high-quality and high-resolution diffusion-weighted imaging datasets. Hum Brain Mapp 32(4):544-563

122. Andrasko J (1976) Water diffusion permeability of human erythrocytes studied by a pulsed gradient NMR technique. Biochim Biophys Acta 428(2):304-311

123. Thelwall PE, Grant SC, Stanisz GJ, Blackband SJ (2002) Human erythrocyte ghosts: exploring the origins of multiexponential water diffusion in a model biological tissue with magnetic resonance. Magn Reson Med 48(4):649-657

124. Benga G, Chapman BE, Kuchel PW (2009) Comparative NMR studies of diffusional water permeability of red blood cells from different species: XV. Agile wallaby (Macropus agilis), rednecked wallaby (Macropus rufogriseus) and Goodfellow's tree kangaroo (Dendrolagus goodfellowi). Comp Biochem Physiol A Physiol 154(1):105-109

125. Lisitza NV, Warren WS, Song Y-Q (2007) Study of diffusion in erythrocyte suspension using internal magnetic field inhomogeneity. J Magn Reson 187(1):146-154

126. Zhao L, Sukstanskii AL, Kroenke CD, Song J, Piwnica-Worms D, Ackerman JJH, Niel JJ (2008) Intracellular Water Specific MR of Microbead-Adherent Cells: HeLa Cell Intracellular Water Diffusion. Magn Reson Med 59:79-84

127. Åslund I, Topgaard D (2009) Determination of the self-diffusion coefficient of intracellular water using PGSE NMR with variable gradient pulse length. J Magn Reson 201(2):250-254

128. Sehy JV, Ackerman JJH, Neil JJ (2002) Evidence that both fast and slow water ADC components arise from intracellular space. Magn Reson Med 48(5):765-770
129. Galons J-P, Lope-Piedrafita S, Divijak JL, Corum C, Gillies RJ, Trouard TP (2005) Uncovering of intracellular water in cultured cells. Magn Reson Med 54(1):79-86

130. Cooper RL, Chang DB, Young AC, Martin CJ, Ancker-Johnson D (1974) Restricted diffusion in biophysical systems. Experiment. Biophys J 14(3):161-177

131. Tanner JE (1979) Self diffusion of water in frog muscle. Biophys J 28(1):107-116

132. Seo Y, Morita Y, Kusaka Y, Steward MC, Murakami M (1996) Diffusion of water in rat sciatic nerve measured by $1 \mathrm{H}$ pulsed field gradient NMR: compartmentation and anisotropy. Jpn J Physiol 46(2):163-169

133. Assaf Y, Cohen Y (2000) Assignment of the water slow-diffusing component in the central nervous system using q-space diffusion MRS: implications for fiber tract imaging. Magn Reson Med 43(2):191-199

134. Chin C-L, Wehrli FW, Fan Y, Hwang SN, Schwartz ED, Nissanov J, Hackney DB (2004) Assessment of axonal fiber tract architecture in excised rat spinal cord by localized NMR q-space imaging: simulations and experimental studies. Magn Reson Med 52(4):733-740

135. Ong HH, Wright AC, Wehrli SL, Souza A, Schwartz ED, Hwang SN, Wehrli FW (2008) Indirect measurement of regional axon diameter in excised mouse spinal cord with q-space imaging: simulation and experimental studies. Neuroimage 40(4):1619-1632

136. Bar-Shir A, Cohen Y (2008) High b-value q-space diffusion MRS of nerves: structural information and comparison with histological evidence. NMR Biomed 21(2):165-174

137. Biton IE, Duncan ID, Cohen Y (2007) q-Space diffusion of myelin-deficient spinal cords. Magn Reson Med 58(5):993-1000

138. Ronen I, Moeller S, Ugurbil K, Kim D-S (2006) Analysis of the distribution of diffusion coefficients in cat brain at $9.4 \mathrm{~T}$ using the inverse Laplace transformation. Magn Reson Imaging 24(1):61-68

139. Does MD, Beaulieu C, Allen PS, Snyder RE (1998) Multicomponent $\mathrm{T} 1$ relaxation and magnetisation transfer in peripheral nerve. Magn Reson Imaging 16(9):1033-1041

140. Beaulieu C, Fenrich FR, Allen PS (1998) Multicomponent water proton transverse relaxation and T2-discriminated water diffusion in myelinated and nonmyelinated nerve. Magn Reson Imaging 16(10):1201-1210

141. Whittall KP, MacKay AL, Graeb DA, Nugent RA, Li DK, Paty DW (1997) In vivo measurement of T2 distributions and water contents in normal human brain. Magn Reson Med 37(1):34-43

142. Laule C, Vavasour IM, Kolind SH, Li DKB, Traboulsee TL, Moore GRW, MacKay AL (2007) Magnetic resonance imaging of myelin. Neurotherapeutics 4(3):460-484

143. Deoni SCL, Rutt BK, Arun T, Pierpaoli C, Jones DK (2008) Gleaning multicomponent $\mathrm{T} 1$ and $\mathrm{T} 2$ information from steadystate imaging data. Magn Reson Med 60(6):1372-1387

144. Does MD, Snyder RE (1995) T2 relaxation of peripheral nerve measured in vivo. Magn Reson Imaging 13(4):575-580

145. Mulkern RV, Zengingonul HP, Robertson RL et al (2000) Multicomponent apparent diffusion coefficients in human brain: relationship to spin-lattice relaxation. Magn Reson Med 44(2):292-300

146. Pfeuffer J, Provencher SW, Gruetter R (1999) Water diffusion in rat brain in vivo as detected at very large $b$ values is multicompartmental. Magn Reson Mater Phy 8(2):98-108

147. Huisman TAGM, Loenneker T, Barta G, Bellemann ME, Hennig J, Fischer JE, Il'yasov KA (2006) Quantitative diffusion tensor MR imaging of the brain: field strength related variance of apparent diffusion coefficient (ADC) and fractional anisotropy (FA) scalars. Eur Radiol 16(8):1651-1658

148. Mulkern RV, Gudbjartsson H, Westin C-F et al (1999) Multicomponent apparent diffusion coefficients in human brain. NMR Biomed 12(1):51-62 
149. Maier SE, Vajapeyam S, Mamata H, Westin C-F, Jolesz FA, Mulkern RV (2004) Biexponential diffusion tensor analysis of human brain diffusion data. Magn Reson Med 51(2):321-330

150. Clark CA, Hedehus M, Moseley ME (2002) In vivo mapping of the fast and slow diffusion tensors in human brain. Magn Reson Med 47(4):623-628

151. Syková E, Nicholson C (2008) Diffusion in brain extracellular space. Physiol Rev 88(4):1277-1340

152. Chin C-L, Wehrli FW, Hwang SN, Takahashi M, Hackney DB (2002) Biexponential diffusion attenuation in the rat spinal cord: computer simulations based on anatomic images of axonal architecture. Magn Reson Med 47(3):455-460

153. Jespersen SN, Kroenke CD, Østergaard L, Ackerman JJH, Yablonskiy DA (2007) Modeling dendrite density from magnetic resonance diffusion measurements. Neuroimage 34(4):1473-1486

154. Clark CA, Hedehus M, Moseley ME (2001) Diffusion time dependence of the apparent diffusion tensor in healthy human brain and white matter disease. Magn Reson Med 45(6): $1126-1129$

155. Does MD, Parsons EC, Gore JC (2003) Oscillating gradient measurements of water diffusion in normal and globally ischemic rat brain. Magn Reson Med 49(2):206-215

156. Jones DK (2003) Determining and visualizing uncertainty in estimates of fiber orientation from diffusion tensor MRI. Magn Reson Med 49(1):7-12

157. Barazany D, Basser PJ, Assaf Y (2009) In vivo measurement of axon diameter distribution in the corpus callosum of rat brain. Brain 132(Pt 5):1210-1220

158. Quirk JD, Bretthorst GL, Duong TQ, Snyder AZ, Springer CS, Ackerman JJH, Neil JJ (2003) Equilibrium water exchange between the intra- and extracellular spaces of mammalian brain. Magn Reson Med 50(3):493-499

159. Pfeuffer J, Bröer S, Bröer A, Lechte M, Flögel U, Leibfritz D (1998) Expression of aquaporins in Xenopus laevis oocytes and glial cells as detected by diffusion-weighted $1 \mathrm{H}$ NMR spectroscopy and photometric swelling assay. Biochim Biophys Acta 1448:27-36

160. Does MD, Gore JC (2002) Compartmental study of T(1) and $\mathrm{T}(2)$ in rat brain and trigeminal nerve in vivo. Magn Reson Med 47(2):274-283

161. Vavasour IM, Whittall KP, Li DK, MacKay AL (2000) Different magnetization transfer effects exhibited by the short and long $\mathrm{T}(2)$ components in human brain. Magn Reson Med 44(6): $860-866$

162. Ford JC, Hackney DB (1997) Numerical model for calculation of apparent diffusion coefficients (ADC) in permeable cylinders-comparison with measured ADC in spinal cord white matter. Magn Reson Med 37(3):387-394

163. Hwang SN, Chin C-L, Wehrli FW, Hackney DB (2003) An image-based finite difference model for simulating restricted diffusion. Magn Reson Med 50(2):373-382

164. Tien T, Ottova-Leitmannova A (2000) Membrane transport. In: Tien T, Ottova-Leitmannova A (eds) Membrane biophysics: as viewed from experimental bilayer lipid membranes (Planar lipid bilayers and spherical liposomes), 1st edn. Elsevier Science, Amsterdam, pp 221-282

165. Nilsson M, Hagslätt H, Van Westen D, Wirestam R, Ståhlberg F, Lätt J (2010) A mechanism for exchange between intraaxonal and extracellular water: Permeable nodes of Ranvier. Proc Intl Soc Mag Reson Med 18:1570

166. Sotak CH (2002) The role of diffusion tensor imaging in the evaluation of ischemic brain injury-a review. NMR Biomed 15(7-8):561-569

167. Schwarcz A, Ursprung Z, Berente Z, Bogner P, Kotek G, Meric P, Gillet B, Beloeil J-C, Dóczi T (2007) In vivo brain edema classification: new insight offered by large b-value diffusionweighted MR imaging. J Magn Reson Imaging 25(1):26-31

168. Brugières $\mathrm{P}$, Thomas $\mathrm{P}$, Maraval A, Hosseini $\mathrm{H}$, Combes $\mathrm{C}$, Chafiq A, Ruel L, Breil S, Peschanski M, Gaston A (2004) Water diffusion compartmentation at high $\mathrm{b}$ values in ischemic human brain. Am J Neuroradiol 25(5):692-698

169. Jensen JH, Falangola MF, Hu C, Tabesh A, Rapalino O, Lo C, Helpern JA (2011) Preliminary observations of increased diffusional kurtosis in human brain following recent cerebral infarction. NMR Biomed 24(5):452-457

170. Geijer B, Lindgren A, Brockstedt S, Ståhlberg F, Holtås S (2001) Persistent high signal on diffusion-weighted MRI in the late stages of small cortical and lacunar ischaemic lesions. Neuroradiology 43(2):115-122

171. Yang Q, Tress BM, Barber PA, Desmond PM, Darby DG, Gerraty RP, Li T, Davis SM (1999) Serial study of apparent diffusion coefficient and anisotropy in patients with acute stroke. Stroke 30(11):2382-2390

172. Budde MD, Frank JA (2010) Neurite beading is sufficient to decrease the apparent diffusion coefficient after ischemic stroke. Proc Natl Acad Sci USA 107(32):14472-14477

173. Does MD, Gore JC (2000) Compartmental study of diffusion and relaxation measured in vivo in normal and ischemic rat brain and trigeminal nerve. Magn Reson Med 43(6):837-844

174. Silva MD, Omae T, Helmer KG, Li F, Fisher M, Sotak CH (2002) Separating changes in the intra- and extracellular water apparent diffusion coefficient following focal cerebral ischemia in the rat brain. Magn Reson Med 48(5):826-837

175. Duong TQ, Ackerman JJH, Ying HS, Neil JJ (1998) Evaluation of extra- and intracellular apparent diffusion in normal and globally ischemic rat brain via 19F NMR. Magn Reson Med 40(1):1-13

176. Sundgren PC, Dong Q, Gómez-Hassan D, Mukherji SK, Maly P, Welsh R (2004) Diffusion tensor imaging of the brain: review of clinical applications. Neuroradiology 46(5):339-350

177. Sen PN, Basser PJ (2005) A model for diffusion in white matter in the brain. Biophys J 89(5):2927-2938

178. Assaf Y, Pasternak O (2008) Diffusion tensor imaging (DTI)based white matter mapping in brain research: a review. J Mol Neurosci 34(1):51-61

179. Frank LR (2001) Anisotropy in high angular resolution diffusion-weighted MRI. Magn Reson Med 45(6):935-939

180. Alexander AL, Hasan KM, Lazar M, Tsuruda JS, Parker DL (2001) Analysis of partial volume effects in diffusion-tensor MRI. Magn Reson Med 45(5):770-780

181. Vos SB, Jones DK, Viergever MA, Leemans A (2011) Partial volume effect as a hidden covariate in DTI analyses. Neuroimage 55(4): 1566-1576

182. Jones DK, Knösche TR, Turner R (2012) White matter integrity, fiber count, and other fallacies: the do's and don'ts of diffusion MRI. Neuroimage. doi:10.1016/j.neuroimage.2012.06.081

183. Douaud G, Jbabdi S, Behrens TEJ et al (2011) DTI measures in crossing-fibre areas: increased diffusion anisotropy reveals early white matter alteration in MCI and mild Alzheimer's disease. Neuroimage 55(3):880-890

184. Tournier JD, Calamante F, Gadian DG, Connelly A (2004) Direct estimation of the fiber orientation density function from diffusion-weighted MRI data using spherical deconvolution. Neuroimage 23(3):1176-1185

185. Assaf Y, Ben-Bashat D, Chapman J et al (2002) High b-value q-space analyzed diffusion-weighted MRI: application to multiple sclerosis. Magn Reson Med 47(1):115-126

186. Assaf Y, Mayzel-Oreg O, Gigi A et al (2002) High b value q-space-analyzed diffusion MRI in vascular dementia: a preliminary study. J Neurol Sci 203-204:235-239 
187. Mardor Y, Pfeffer R, Spiegelmann R et al (2003) Early detection of response to radiation therapy in patients with brain malignancies using conventional and high b-value diffusion-weighted magnetic resonance imaging. J Clin Oncol 21(6):1094-1100

188. Van Essen DC, Ugurbil K, Auerbach E et al (2012) The human connectome project: a data acquisition perspective. Neuroimage 62(4):2222-2231
189. Schwarcz A, Bogner P, Meric P, Correze J-L, Berente Z, Pál J, Gallyas F, Doczi T, Gillet B, Beloeil J-C (2004) The existence of biexponential signal decay in magnetic resonance diffusionweighted imaging appears to be independent of compartmentalization. Magn Reson Med 51(2):278-285 